\title{
The BeppoSAX catalog of GRB X-ray afterglow observations ${ }^{\star}$
}

\author{
M. De Pasquale ${ }^{1,2, \star \star}$, L. Piro ${ }^{1}$, B. Gendre ${ }^{1}$, L. Amati ${ }^{3}$, L. A. Antonelli ${ }^{4}$, E. Costa $^{1}$, M. Feroci ${ }^{1}$, \\ F. Frontera ${ }^{5}$, L. Nicastro ${ }^{7}$, P. Soffitta ${ }^{1}$, and J. in't Zand ${ }^{6}$
}

1 INAF Rome, via fosso del cavaliere 100, 00133 Roma, Italy e-mail: mdp@mssl.ucl.ac.uk;

[luigi.piro; bruce.gendre; enrico.costa;marco.feroci;paolo.soffitta]@.iasf-roma.inaf.it

2 Mullard Space Science Laboratory, University College of London, Holmbury St. Mary, Dorking, RH5 6NT, UK

3 INAF - Istituto di Astrofisica Spaziale e Fisica Cosmica, via P. Gobetti 101, 40219 Bologna, Italy e-mail: amati@bo.iasf.cnr.it

4 Rome Astronomical Observatory, via di Frascati 33, 00044 Rome, Italy

e-mail: a. antonelli@mporzio.astro.it

5 Università di Ferrara, via Paradiso 12, 44100 Ferrara, Italy e-mail: frontera@fe.infn.it

6 Space Research Organization of the Netherlands, Sorbonnelaan 2, 3584 CA Utrecht, The Netherlands e-mail: jeanz@sron.nl

7 Istituto Astrofisica Spaziale e Fisica Cosmica, Sezione di Palermo, INAF, via U. La Malfa 153, 90146 Palermo, Italy e-mail: nicastro@pa.iasf.cnr.it

Received 29 July 2005 / Accepted 18 March 2006

\section{ABSTRACT}

\begin{abstract}
We present the catalog of X-ray afterglow observed by BeppoSAX from the launch of the satellite to the end of the mission. Thirtythree X-ray afterglows out of 39 observations were securely identified based on their fading behavior. We have extracted the continuum parameters (decay index, spectral index, flux, absorption) for all available afterglows. We point out a possible correlation between the $\mathrm{X}$-ray afterglow luminosity and the energy emitted during the prompt $\gamma$-ray event. We do not detect a significant jet signature within the afterglows, implying a lower limit on the beaming angle, nor a standard energy release when X-ray fluxes are corrected for beaming. Our data support the hypothesis that the burst should be surrounded by an interstellar medium rather than a wind environment, and that this environment should be dense. This may be explained by a termination shock located near the burst progenitor. We finally point out that some dark bursts may be explained by an intrinsic faintness of the event, while others may be strongly absorbed.
\end{abstract}

Key words. X-rays: general - gamma rays: bursts - catalogs

\section{Introduction}

Discovered in the early 70's (Klebesadel et al. 1973), GammaRay Bursts (GRBs) were a mysterious phenomenon for 25 years. The lack of any optical counterpart prevented observers from determining the distance - galactic or extragalactic - and therefore the amount of energy involved, which was uncertain within 10 orders of magnitude. Therefore, a number of different models were able to explain the observed prompt gamma-ray emission.

The situation changed dramatically with the first fast and precise localization of GRB that was obtained by the BeppoSAX satellite (Piro 1995; Boella et al. 1997) in 1997. This satellite combined a gamma-ray burst monitor (that provided the burst trigger) with X-ray cameras (that were able to asses a precise position and to carry out follow-up observations). This observational strategy led to the discovery of the X-ray (Costa et al. 1997), optical (van Paradijis et al. 1997), and radio (Frail et al. 1997) afterglows. The spectroscopy of the optical counterpart of

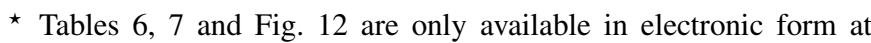
http://www. edpsciences.org

$\star \star$ Present address: Mullard Space Science Laboratory, Holmbury St. Mary, Dorking, Surrey RH5 6NT, UK the burst also allowed the distance of these events to be firmly established as cosmological (Metzger et al. 1997).

With the end of the BeppoSAX mission (April 2002) and its reentry, a page of the GRB afterglow study was turned, but the observations remained within the archives. To prepare for the future, we have initiated a complete re-analysis of all the X-ray observations that have been done. In this first paper, we present the legacy of BeppoSAX: its X-ray afterglow catalog, focusing on the continuum properties. We will also compare our results with those of previous studies on GRB X-ray afterglows (Frontera et al. 2003; Piro 2004). A second paper (Gendre et al. 2006) will describe the XMM-Newton and Chandra catalog of X-ray afterglows. In a forthcoming paper (Gendre et al., in preparation), we will discuss a systematic study of line emission in the X-ray afterglow spectra.

This article is organized as follows. In Sect. 2 we present the data analysis and the results. We discuss these results in Sect. 3 in the light of the fireball model. We investigate the so-called Dark Burst phenomenon in Sect. 4, before concluding.

\section{Data reduction and analysis}

BeppoSAX simultaneously detected and localized 51 GRBs in the Gamma Ray Burst Monitor (GRBM, Frontera et al. 1997) 
and Wide Field Cameras (WFC, Jager et al. 1997) within its sixyear lifetime (Frontera et al. 2004) . These bursts have been included in our analysis sample. We note that this set is biased against X-ray rich GRBs and especially X-ray flashes (Heise et al. 2002), i.e., bursts with weak or absent signals in the GRBM and normal counterparts in the WFC. In our sample, we also included GRB 991106, GRB 020410, and GRB 020427, although they gave no detection in the $\mathrm{GRBM}^{1}$, due to the fact that a subsequent observation with the BeppoSAX narrow field instruments was performed after the localization with the WFC. Data on these bursts are reported in Tables 6 and 7. We used only bursts discovered at the onset of the prompt emission (i.e., not including late archive re-analysis), as these are the only ones for which an afterglow follow-up observation was possible.

Overall, it was possible to follow up 37 bursts with the narrow field instruments. One other afterglow observation (GRB 000926) was carried out following external triggers. Finally, in the case of GRB 980703, BeppoSAX detected the burst while it was outside the WFC field of view, and the follow-up observation was performed on the basis of a localization by the RXTE All Sky Monitor. In this paper, we present the data gathered by the Narrow Field Instruments (NFI) Low Energy Concentrator Spectrometer (LECS, 0.1-10 keV, Parmar et al. 1997) and Medium Energy Concentrator Spectrometer (MECS, 1.6-10 keV, Boella et al. 1997). The first of this sample (GRB 960720) was followed up late, while 38 had fast (within $1 \mathrm{day}^{2}$ ) follow-up observations. We analyzed 37 of these fast follow-up observations, excluding GRB 990705, strongly contaminated by nearby X-ray source.

A typical observation starts $\sim 8-9 \mathrm{~h}$ after the burst and its duration is about $1 \times 10^{5} \mathrm{~s}$ for MECS and $7 \times 10^{4}$ for LECS. The net exposure lasts $\sim 1 / 2$ of the observation for MECS and $1 / 4$ for LECS.

\subsection{Afterglow identification and temporal analysis}

The first step of data analysis is the source detection, to find the afterglow. For this purpose, we used the MECS data because this instrument has a higher sensitivity than that of the LECS. We extracted the image, ran the detection tool within Ximage version $4.3^{3}$ on it and selected all the sources with at least a $3 \sigma$ significance located inside the WFC error box. In the special cases of GRB 980703 and GRB 000926, we used the IPN error box (Hurley et al. 2000) and ASM error box (Levine et al. 1998), respectively, as these bursts were outside the WFC field of view. The afterglow was recognized by its fading behavior. The light curves were generated from counts extracted within a circle area centered on the source with a radius of 4 arcmin. We chose this value because $\gtrsim 90 \%$ of the source counts are within this region (Fiore et al. 1999). We also selected counts between the 1.6 and $10 \mathrm{keV}$ interval, which is the optimal range of work for the MECS.

The associated background was extracted using an annulus centered at the same position as the source extraction region, with inner and outer radii of 4.5 and 10 arcmin, respectively. To take into account the effects of effective area variation and the

\footnotetext{
1 In the case of GRB 020410, GRBM was actually switched off at the time of the burst. A $\gamma$-ray signal was detected by Konus (Nicastro et al. 2004).

22 days for GRB 000926.

3 See http://heasarc.gsfc.nasa.gov/docs/xanadu/ ximage/ximage.html
}

shadow caused by the MECS instrument support, we normalized the count number observed within the annulus by a factor determined from the count number observed within the same regions of the library backgrounds.

We used the local background rather than the library background for light curves to take into account any possible time fluctuation. We developed an IDL script to construct and fit light curves. This algorithm can calculate adequate errors even in the case of few counts per bin by using a Poissonian statistics. However, if possible, the width of temporal bins was chosen wide enough to have at least 15-20 counts/bin (background subtracted), to apply a proper Gaussian fit (see below). When available, subsequent TOOs were also used to better constrain the light curve behavior.

The light curves were fitted with a simple power law model, using the Levenberg-Marquardt method to minimize the $\chi^{2}$ statistic. Thirty-one sources had a positive decaying index (in the following, we used the convention $F_{\mathrm{X}} \propto t^{-\delta}$, thus a decaying source has a positive decay index) at the $90 \%$ confidence level. These sources were identified as the X-ray afterglow of each burst $^{4}$. For three of these sources (GRB 971227, GRB 990217, and GRB 000529) the decay index value is positive but not well constrained. We report the decay indexes we obtained for all these 31 sources (henceforth, all errors reported are at $1 \sigma$, while upper limits are quoted at the $90 \%$ confidence level, unless otherwise specified) in Table 1.

In three cases (GRB 970111, GRB 991106, and GRB 000615), we detected only one source that did not display any significant fading behavior within the WFC error box. We refer to these as candidate afterglows. We have calculated the probability of observing a serendipitous source at the observed flux level within the WFC error box for these 3 bursts, adopting the $\log N-\log S$ distribution for BeppoSAX released by Giommi et al. (2000). The probabilities are $\cong 0.027$ for GRB 000615 and $\cong 0.05$ for GRB 970111 and GRB 991106 . The probability that all of these 3 sources are not afterglows is $\sim 10^{-4}$. We note, however, that these probabilities have been calculated for extragalactic sources; for low Galactic latitude events like GRB $991106\left(b \simeq-3^{\circ}\right)$, the value may differ significantly. Cornelisse et al. (2002) indicated that GRB 991106 could in fact be a Galactic type-I X-ray burster.

In two cases (GRB 010220 and GRB 020321), we did not detect any source with $3 \sigma$ significance within the WFC error box. We report in Table 1 the $3 \sigma$ detection upper limits.

Some observations deserve special comments. GRB 990907 was observed for $\sim 1000$ s only and no decaying behavior can be detected within the light curve of the source found inside the WFC error box. However, given the high flux of this source $\left(\sim 10^{-12} \mathrm{erg} \mathrm{cm}^{-2} \mathrm{~s}^{-1}\right.$ in the $1.6-10 \mathrm{keV}$ band), the probability of having observed a serendipitous source was $\sim 10^{-3}$. We have thus assumed that this source was indeed the X-ray afterglow of GRB 990907. In the case of GRB 980425, we analyzed the source S1 coincident with SN1998bw (Pian et al. 1999). We do not include it in the following discussion as the detected X-ray emission could be strongly affected by SN1998bw.

\section{We present the light curves in Fig. 12.}

\footnotetext{
${ }^{4}$ In the cases of GRB 000926 and GRB 020427, we have used data gathered by the Chandra X-ray observatory to constrain the decay index (see Piro et al. 2001; Gendre et al. 2006). For GRB 011121, we have used the last WFC data points (see Piro et al. 2005).
} 
Table 1. Properties of the X-ray afterglows detected by BeppoSAX. We indicate the absorbed flux extrapolated or interpolated to $11 \mathrm{~h}$ after the burst, the temporal decay and the energy spectral index, the Galactic column density from Dickey \& Lockman (1990), the excess of absorption at $z=0$, and the excess of absorption at the proper redshift of the burst (assuming a distance of $z=1$ when the host galaxy redshift was unknown).

\begin{tabular}{|c|c|c|c|c|c|c|}
\hline GRB name & $\begin{array}{c}1.6-10 \mathrm{keV} \text { Flux } \\
\left(10^{-13} \mathrm{erg} \mathrm{cm}^{-2} \mathrm{~s}^{-1}\right)\end{array}$ & $\begin{array}{c}\text { Decay } \\
\text { index } \\
\delta\end{array}$ & $\begin{array}{c}\text { Spectral } \\
\text { index } \\
\alpha\end{array}$ & $\begin{array}{c}\text { Galactic } \\
\text { column } \\
\text { density } \\
\left(10^{22} \mathrm{~cm}^{-2}\right)\end{array}$ & $\begin{array}{c}\text { Excess of } \\
\text { column } \\
\text { density }(z=0) \\
\left(10^{22} \mathrm{~cm}^{-2}\right)\end{array}$ & $\begin{array}{l}\text { Excess of } \\
\text { column } \\
\text { density } \\
\left(10^{22} \mathrm{~cm}^{-2}\right)\end{array}$ \\
\hline GRB 970111 & $0.75 \pm 0.47$ & $2.8^{-3.7}$ & - & - & - & - \\
\hline GRB 970228 & $20.8 \pm 2.7$ & $1.32_{-0.20}^{+0.15}$ & $1.04_{-0.27}^{+0.21}$ & 0.16 & $<0.4$ & $<1.12$ \\
\hline GRB 970402 & $1.35 \pm 0.73$ & $1.11_{-0.56}^{+1.5}$ & - & - & - & - \\
\hline GRB 970508 & $5.72 \pm 0.90$ & $0.80_{-0.15}^{+0.18}$ & $1.40_{-0.27}^{+0.32}$ & 0.05 & $0.67_{-0.35}^{+0.73}$ & $2.63_{-1.37}^{+2.5}$ \\
\hline GRB 971214 & $6.36 \pm 0.91$ & $1.00 \pm 0.22$ & $1.08_{-0.23}^{+0.40}$ & 0.02 & $<1.23$ & $<53$ \\
\hline GRB 971227 & - & $>0.4$ & - & - & - & - \\
\hline GRB 980329 & $6.00 \pm 0.56$ & $1.42_{-0.48}^{+0.62}$ & $1.44_{-0.26}^{+0.32}$ & 0.09 & $<0.5$ & $<3.07$ \\
\hline GRB 980425 & $2.82 \pm 0.59$ & $0.10 \pm 0.06$ & - & - & - & - \\
\hline GRB 980515 & $5.6 \pm 2.2$ & $>0.51$ & - & - & - & - \\
\hline GRB 980519 & $3.9_{-1.1}^{+1.2}$ & $2.18_{-0.65}^{+0.89}$ & $2.43_{-0.65}^{+0.97}$ & 0.17 & $0.97_{-0.65}^{+1.13}$ & $5.1_{-3.8}^{+6.0}$ \\
\hline GRB 980613 & $2.6_{-1.1}^{+1.1}$ & $1.49_{-0.96}^{+1.9}$ & - & - & - & - \\
\hline GRB 980703 & $14.0_{-3.2}^{+7.1 .0}$ & $1.10_{-0.28}^{+0.86}$ & $1.71 \pm 0.29$ & 0.06 & $0.52_{-0.25}^{+0.38}$ & $2.6_{-1.3}^{+2.0}$ \\
\hline GRB 981226 & $2.8_{1.3}^{2.1 .2}$ & $0.66_{-0.44}^{+0.68}$ & - & - & - & - \\
\hline GRB 990123 & $54.2 \pm 1.7$ & $1.45 \pm 0.06$ & $0.99 \pm 0.05$ & 0.02 & $0.013_{-0.008}^{+0.01}$ & $0.10_{-0.06}^{+0.08}$ \\
\hline GRB 990217 & $2.8_{-1.4}^{+5.1}$ & $>0$ & - & - & - & - \\
\hline GRB 990510 & $34.7 \pm 2.1$ & $1.4 \pm 0.1$ & $1.17 \pm 0.09$ & 0.09 & $<0.11$ & $<0.93$ \\
\hline GRB 990627 & $3.3_{-1.5}^{+1.6}$ & $1.32_{-0.92}^{+1.7}$ & - & - & - & - \\
\hline GRB 990704 & $5.87 \pm 0.84$ & $0.88_{-0.20}^{+0.28}$ & $1.68_{-0.38}^{+0.45}$ & 0.03 & $0.72_{-0.4}^{+0.7}$ & $4.1_{-2.3}^{+3.4}$ \\
\hline GRB 990806 & $3.20 \pm 0.87$ & $0.9_{-0.42}^{+0.47}$ & $1.31_{-0.43}^{+0.57}$ & 0.04 & $<2.2$ & $<13.15$ \\
\hline GRB 990907 & $10.6 \pm 4.0$ & - & - & - & - & - \\
\hline GRB 991014 & $5.4_{-1.5}^{+1.9}$ & $1.10_{-0.32}^{+0.50}$ & - & - & - & - \\
\hline GRB 991106 & $1.26 \pm 0.47$ & $1.1_{-2.1}^{+2.52}$ & - & - & - & - \\
\hline GRB 000210 & $3.10_{-0.96}^{+0.90}$ & $1.41_{-0.77}^{+0.98}$ & $1.54_{-0.4}^{+0.31}$ & 0.03 & $0.4_{-0.23}^{+0.4}$ & $1.71_{-1.01}^{+1.7}$ \\
\hline GRB 000214 & $6.2_{-1.8}^{+2.1}$ & $0.68 \pm 0.41$ & $1.04 \pm 0.27$ & 0.06 & $<0.17$ & $<0.36$ \\
\hline GRB 000528 & $3.0_{-1.4}^{+4.1}$ & $0.8_{-1.5}^{+0.5}$ & - & - & - & - \\
\hline GRB 000529 & $1.6 \pm 1.2$ & $>0$ & - & - & - & - \\
\hline GRB 000615 & $1.28 \pm 0.38$ & $-0.23_{-0.94}^{+1.4}$ & - & - & - & - \\
\hline GRB 000926 & $32.6_{-8.7}^{+15.7}$ & $1.79_{-0.16}^{+0.21}$ & - & - & - & - \\
\hline GRB 001109 & $23.2_{-4.5}^{+5.8}$ & $1.47_{-0.27}^{+0.10}$ & $1.29_{-0.26}^{+0.27}$ & 0.04 & $0.62_{-0.32}^{+0.45}$ & $3.4_{-17}^{+2.3}$ \\
\hline GRB 010214 & $3.06_{-0.64}^{+0.71}$ & $1.90_{-0.53}^{+0.91}$ & - & - & - & - \\
\hline GRB 010220 & $<1.43$ & - & - & - & - & - \\
\hline GRB 010222 & $70.6 \pm 3.4$ & $1.35 \pm 0.06$ & $1 \pm 0.06$ & 0.02 & $0.16 \pm 0.04$ & $1.27_{-0.31}^{+0.33}$ \\
\hline GRB 011121 & $13.6 \pm 1.5$ & $1.30 \pm 0.03$ & - & - & - & - \\
\hline GRB 020321 & $<3.4$ & - & - & - & - & - \\
\hline GRB 020322 & $3.8 \pm 0.8$ & $0.84_{-0.35}^{+0.46}$ & - & - & - & - \\
\hline GRB 020410 & $77.8_{-6.9}^{+6.3}$ & $0.92 \pm 0.12$ & $1.3 \pm 0.19$ & 0.08 & $<0.7$ & $<4.8$ \\
\hline GRB 020427 & $4.8 \pm 1.7$ & $1.3_{-0.12}^{+0.10}$ & - & - & - & - \\
\hline
\end{tabular}

\subsection{Spectral analysis}

The X-ray afterglow spectra have been accumulated from the LECS and MECS during the first TOO only, for those afterglows with more than 150 photons in the MECS (background subtracted). 15 GRBs passed this criterion; their spectra are presented in Fig. 1.

We have generally collected LECS counts within a circle centered on the source with radius $r=8$ arcmin, which again encircles $>90 \%$ of source counts. LECS data were restriced to the $0.1-4.0 \mathrm{keV}$ band, where the response matrix is more accurate. As for MECS, we collected counts with the same criteria we applied for the time analysis.

For spectral analysis, we used the library spectral backgrounds for both LECS and MECS as they have a very good signal-to-noise ratio, since they have been exposed for a long time $^{5}$. However, the library backgrounds have been taken at high Galactic latitudes, with an average Galactic absorption around $2-3 \times 10^{20}$. Several afterglows in our sample have been observed in fields with an absorption much higher than this value. For these bursts, the local background would differ from the library one at low energy (e.g., below $0.3 \mathrm{keV}$ ). The use of the library background at low energy would result in an underestimate of the low-energy signal and, consequently, in an overestimate of the intrinsic absorbing column of the burst. Therefore, to solve this problem, we have increased the minimum energy for LECS to $0.4 \mathrm{keV}$, when the Galactic column density was

\footnotetext{
${ }^{5}$ In the case of GRB 970111 and GRB 970402, better results were obtained by using local background.
} 


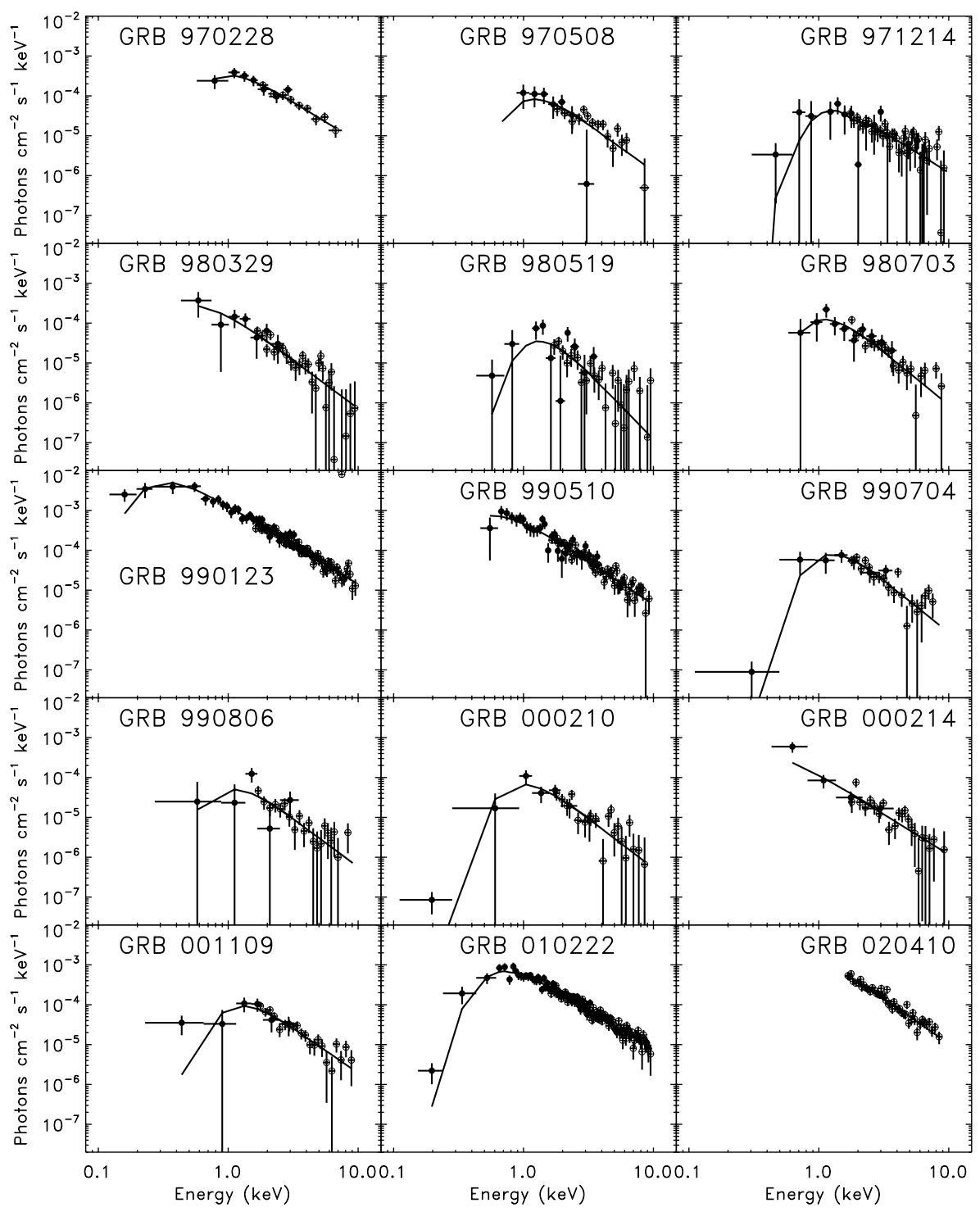

Fig. 1. X-ray spectra of the afterglows observed by BeppoSAX. Filled circles represent LECS data points and open circles represent MECS data points. Note that no LECS information was available for GRB 020410.

larger than $5 \times 10^{20} \mathrm{~cm}^{-2}$. The spectral analysis was performed in a similar fashion to the time analysis, by requiring at least 15-20 counts/bin.

The standard model to fit the spectral data consists of a constant, a Galactic absorption, an extragalactic absorption (i.e., in situ), and a power law. The constant has been included because of the cross-calibration uncertainties between the LECS and MECS instruments. Its value is obtained in each case by fitting LECS and MECS data in the 1.6-4 keV interval (to reduce absorption effects) with a simple power law model. We find that this constant is usually comprised between 0.6 and 0.9 , in good agreement with previous results of Stratta et al. (2004).

In our work, we calculated the $1.6-10 \mathrm{keV}$ flux of X-ray afterglows $11 \mathrm{~h}$ after the burst trigger. We chose this time to avoid effects of changes in the decaying slope. The average count rate in the MECS has been associated with the average flux given by the spectrum. We took the count rate at $11 \mathrm{~h}$, which is given by the light curves, to compute the flux at that time. In most cases, observations include it. In a few cases (e.g., GRB 000926) the flux was extrapolated.
For those afterglows with less than 150 counts (and thus no spectral analysis), we used a canonical model with a power law energy index of $\alpha=1.2$ (which is typical of X-ray afterglow spectra) to convert the count rate $11 \mathrm{~h}$ after the trigger to the corresponding flux.

All the results of our X-ray afterglow analysis are summarized in Table 1. In Table 2, we report previous results, mostly taken from the review of Frontera et al. (2004). We can see a general agreement of the previous results with ours.

The sample of X-ray afterglows with known redshift observed by BeppoSAX is not very large. We have thus decided to increase it by adding all bursts detected by BeppoSAX, not observed by the BeppoSAX NFI, observed by XMM-Newton or Chandra, and with a known redshift. Browsing the XMM-Newton and Chandra catalog (Gendre et al. 2006), we added GRB 011211 to this sample. This burst, observed by XMM-Newton, presented some evidence for deviation from the simple power law continuum in the spectra (Reeves et al. 2002). While this work has been put into question by some authors (e.g. Borozdin \& Trudolyubov 2003), this deviation could bias the results for this burst. However, we use the results of 
Table 2. Results reported from previous analysis of X-ray afterglows detected by BeppoSAX. In this table, we indicate the temporal decay, the energy index, the fitted value of $n_{\mathrm{H}}$ compared to the galactic column density and the associated reference. A label "W" close to the decay index indicates that this value was obtained by means of WFC and NFI data.

\begin{tabular}{|c|c|c|c|c|c|}
\hline $\begin{array}{l}\text { GRB } \\
\text { name }\end{array}$ & $\begin{array}{c}\text { Temporal } \\
\text { index }^{a} \\
\delta\end{array}$ & $\begin{array}{c}\text { Energy } \\
\text { index } \\
\alpha\end{array}$ & $\begin{array}{c}n_{\mathrm{H}} / n_{\mathrm{H}}^{G} \\
\left(\times 10^{21} \mathrm{~cm}^{-1}\right)\end{array}$ & $\begin{array}{c}\text { 2-10 keV flux } \\
\text { at } 10^{5} \mathrm{~s}^{a} \\
\left(\mathrm{erg} \mathrm{cm}^{-2} \mathrm{~s}^{-1}\right)\end{array}$ & Ref. \\
\hline GRB 970111 & $>1.5$ & - & - & $<1.0 \times 10^{-13}$ & Feroci et al. (1998) \\
\hline GRB 970228 & $1.3 \pm 0.2$ & $1.1 \pm 0.3$ & $3.5_{-2.3}^{+3.3} / 1.6$ & $\sim 6.8 \times 10^{-13}$ & Costa et al. (1997); Frontera et al. (1998) \\
\hline GRB 970402 & $1.45 \pm 0.15$ & $1.7 \pm 0.6$ & $<20 / 2.0$ & $\sim 4.5 \times 10^{-14}$ & Nicastro et al. (1998) \\
\hline GRB 970508 & $1.1 \pm 0.1^{b}$ & $0.5 \pm 0.55$ & $6.0_{-3.3}^{+7.9} / 0.5$ & $3.5 \times 10^{-13}$ & Piro et al. $(1998 b, 1999)$ \\
\hline GRB 971214 & $\sim 1.2$ & $0.6 \pm 0.2$ & $1.0_{-1.0}^{+2.3} / 0.6$ & - & Dal Fiume et al. (2000) \\
\hline GRB 971227 & $1.12_{-0.05}^{+0.08}(\mathrm{~W})$ & [1.1] & {$[0.13] / 0.13$} & $\sim 1.4 \times 10^{-13}$ & Antonelli et al. (1999) \\
\hline GRB 980329 & $1.3 \pm 0.03(\mathrm{~W})$ & $1.4 \pm 0.4$ & $10 \pm 4 / 0.9$ & $2.0 \times 10^{-13}$ & in't Zand et al. (1998) \\
\hline GRB 980425 & $0.16 \pm 0.04$ & $1.0 \pm 0.18$ & {$[0.39] / 0.39$} & $\sim 4.0 \times 10^{-13}$ & Pian et al. (2000) \\
\hline GRB 980519 & $1.83 \pm 0.30$ & $1.8_{-0.5}^{+0.6}$ & $3-20 / 1.73$ & $8.0 \times 10^{-14}$ & Nicastro et al. (1999) \\
\hline GRB 980613 & $1.19 \pm 0.17(\mathrm{~W})$ & - & - & $\sim 2.3 \times 10^{-13}$ & Soffitta et al. (2002) \\
\hline GRB 980703 & $>0.91$ & $1.51 \pm 0.32$ & $36_{-13}^{+22 c} / 0.34$ & $4.5 \times 10^{-13}$ & Vreeswijk et al. (1999) \\
\hline GRB 981226 & $1.3_{-0.4}^{+0.5}$ & $0.92 \pm 0.47$ & {$[0.18] / 0.18$} & $\sim 2.0 \times 10^{-13}$ & Frontera et al. (2000b) \\
\hline GRB $990123^{d}$ & $1.46 \pm 0.04$ & $0.94 \pm 0.08$ & $0.9_{0.9}^{+15} / 0.21$ & $1.25 \times 10^{-12}$ & Maiorano et al. (2005) \\
\hline GRB 990510 & $1.42 \pm 0.07$ & $1.03 \pm 0.08$ & $2.1 \pm 0.6 / 0.94$ & $9.6 \times 10^{-13}$ & Kuulkers et al. (2000) \\
\hline GRB 990704 & $0.83 \pm 0.16$ & $0.7_{-0.2}^{+0.4}$ & {$[0.3] / 0.3$} & $\sim 3.3 \times 10^{-13}$ & Feroci et al. (2001) \\
\hline GRB 990705 & $1.58 \pm 0.06$ & - & - & $<1.2 \times 10^{-13}$ & Frontera et al. (2003) \\
\hline GRB 990806 & $1.15 \pm 0.03(\mathrm{~W})$ & $1.16_{-0.37}^{+0.3}$ & {$[0.35] / 0.35$} & $\sim 2.0 \times 10^{-13}$ & Montanari et al. (2002) \\
\hline GRB 991014 & $>0.4$ & $0.53 \pm 0.25$ & {$[2.5] / 2.5$} & $\sim 3.0 \times 10^{-13}$ & in't Zand et al. (2000b) \\
\hline GRB 000210 & $1.38 \pm 0.03(\mathrm{~W})$ & $0.75 \pm 0.3$ & $<4 \times 10^{21}$ & $\sim 2 \times 10^{-13}$ & Piro et al. (2002) \\
\hline GRB 000214 & $0.8 \pm 0.3$ & $1.0 \pm 0.18$ & $0.7_{-0.7}^{+7.5} / 0.55$ & $\sim 3.5 \times 10^{-13}$ & Antonelli et al. (2000) \\
\hline GRB 000926 & $1.89_{-0.19}^{+0.16 e}$ & $0.9 \pm 0.42$ & $4 / 0.27^{e}, f$ & $9.0 \times 10^{-13}$ & Piro et al. (2001) \\
\hline GRB 001109 & $1.18 \pm 0.05$ & $1.4 \pm 0.3$ & $8.7 \pm 0.4 / 0.42$ & $\sim 8.0 \times 10^{-13}$ & Amati et al. (2003) \\
\hline GRB 010214 & $2.1_{-1.0}^{+0.6}$ & $0.3_{-0.6}^{+0.8}$ & {$[0.27] / 0.27$} & - & Guidorzi et al. (2003) \\
\hline GRB 010222 & $1.33 \pm 0.04$ & $0.97 \pm 0.05$ & $1.5 \pm 0.3 / 0.16$ & $2.4 \times 10^{-12}$ & in't Zand et al. (2001) \\
\hline GRB 011121 & $1.29 \pm 0.03(\mathrm{~W})$ & $1.6 \pm 0.5$ & $<100 /$ & $\sim 10^{-13}$ & Piro et al. (2005) \\
\hline GRB 020321 & - & - & - & $<3 \times 10^{-13}$ & in't Zand et al. (2004) \\
\hline GRB 020410 & $0.81 \pm 0.07$ & $1.05 \pm 0.08$ & - & $\sim 3.5 \times 10^{-12}$ & Nicastro et al. (2004) \\
\hline GRB 020427 & $1.3_{0.09}^{+0.13}$ & $1_{-1.1}^{+2.2}$ & $0.29 / 0.29$ & $\sim 10^{-13}$ & Amati et al. (2004) \\
\hline
\end{tabular}

${ }^{a}$ All upper limits are $3 \sigma$, except for GRB 990705, which are $2 \sigma .{ }^{b}$ From $6 \times 10^{4} \mathrm{~s}$ to $5.8 \times 10^{5} \mathrm{~s} .{ }^{c} n_{\mathrm{H}}$ value corrected for redshift. ${ }^{d}$ Spectral data of the first $20000 \mathrm{~s}$. The time decaying index includes the whole NFI TOO. ${ }^{e}$ SAX plus CHANDRA data (Piro et al. 2001). ${ }^{f}$ Corrected for redshift (Piro et al. 2001). This $n_{\mathrm{H}}^{z}$ value was added to the Galactic column density $n_{\mathrm{H}}^{G}$.

Gendre et al. (2006) obtained with the most updated calibration database. The study of X-ray lines in that spectrum (Gendre et al., in preparation) has shown that the effect on the continuum is small and covered by the given spectral index error bar $(1.3 \pm 0.1)$.

\section{Results and discussion}

\subsection{General properties of $X$-ray afterglows}

We detect an X-ray afterglow in 31 of 36 cases. This constitutes $86 \%$ of the sample. If all doubtful sources are considered to be afterglows, then the fraction of X-ray afterglows increases up to $94 \%$.

In Fig. 2, we present the distribution of the X-ray afterglow flux $F_{\mathrm{X}}$ in the $1.6-10 \mathrm{keV}$ band. It spans approximately 2 orders of magnitude. GRB 020410 afterglow is the object with the highest flux, $\sim 8 \times 10^{-12} \mathrm{erg} \mathrm{cm}^{-2} \mathrm{~s}^{-1}$, while the weakest is 970402 , $\sim 10^{-13} \mathrm{erg} \mathrm{cm}^{-2} \mathrm{~s}^{-1}$. The fit of this distribution with a Gaussian provides a logarithmic mean and width of $\left\langle F_{X}\right\rangle-12.2 \pm 0.1$ and $\sigma_{F \mathrm{x}}=0.5$, respectively. One may wonder if some faint afterglows could be missed due to the detection limit (either due to a low luminosity or to a large distance). In this case, the true

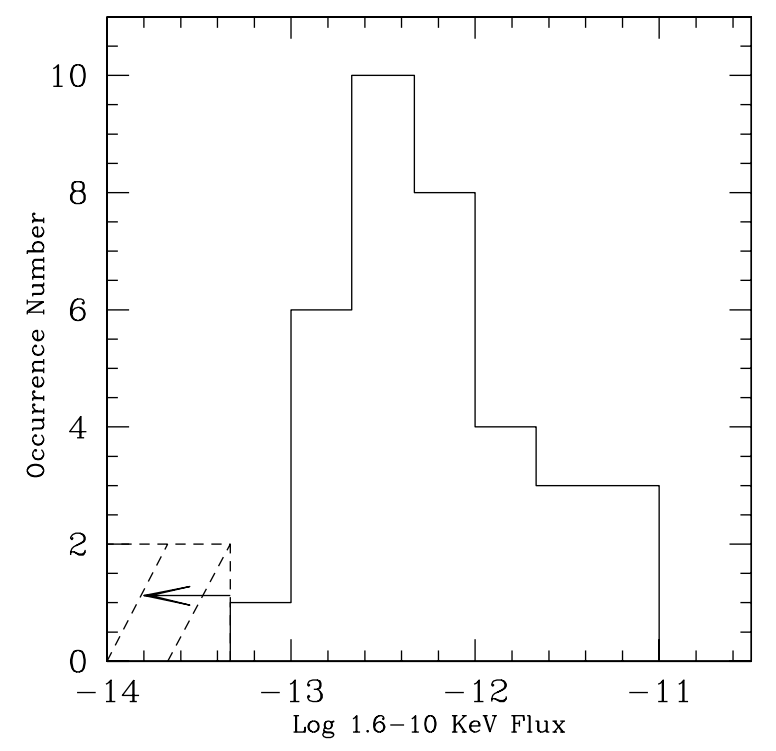

Fig. 2. The distribution of 1.6-10 keV fluxes in the BeppoSAX GRB afterglow sample. All fluxes are indicated $11 \mathrm{~h}$ after the burst. Upper limits have been set to $10^{-13}$ for clarity. 

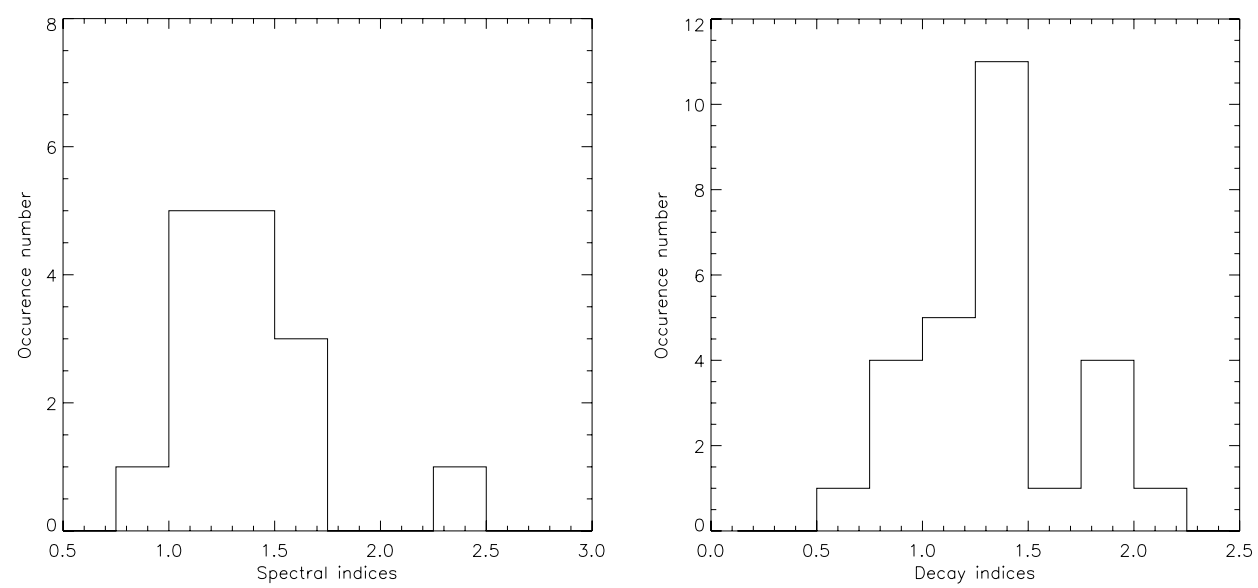

Fig. 3. Left: distribution of the spectral indexes of the afterglow of the BeppoSAX bursts. Right: distribution of the decay indexes of the afterglow of the BeppoSAX bursts.

distribution could be broader than the one we measured. However, the fact that we detect X-ray afterglows in $\sim 90 \%$ of the follow-up observations indicates that this is not the case.

We have also estimated the distribution of the spectral and decay indexes (Fig. 3). The values we have obtained for those parameters are the result of the convolution of the intrinsic distribution with the measurement error. Under the assumption that both are Gaussian, it is possible to deconvolve the two distributions. We have adopted a maximum likelihood method (see De Pasquale et al. 2003; Maccacaro et al. 1988) to gather the best estimates of the parent distribution in the BeppoSAX sample. We have obtained a mean value of $\alpha=1.2 \pm 0.1$ with a width of $0.13_{-0.05}^{+0.11}$ from the spectral index distribution and a mean value of $\delta=1.3 \pm 0.1$ with a width of $0.3 \pm 0.1$ from the decay index distribution. These values depend on the value of $p$, the energy power law index of the electrons which radiate by synchrotron emission within the fireball, and the state of the fireball itself (fast/slow cooling, position of the cooling frequency, beaming, surrounding medium). In Sect. 3.4 we will show that the average properties of the afterglow are consistent with a cooling frequency below the X-ray range. In this case, following Sari et al. (1998), we can determine an average value for $p=2.4 \pm 0.2$.

\subsection{General properties of the prompt emission and selection effects}

In Table 7, we list the properties of the prompt emission of GRB detected by BeppoSAX, extracted from the literature. Figure 4 displays the distribution of the $\gamma$-ray fluence of the BeppoSAX sample. The fit with a Gaussian provides a mean logarithmic fluence of $S_{\gamma}=-5.31$ and a width of distribution $\sigma_{S \gamma}=0.77^{6}$.

One important question concerns the possible selection effects on the flux of the prompt phase. In Fig. 5, we present the isotropic gamma-ray energy and X-ray energy for events with known redshift, emitted in the $40-700 \mathrm{keV}$ and $2-10 \mathrm{keV}$ bands, respectively (in the GRB cosmological rest frames). They have been calculated by using the k-correction of Bloom et al. (2001), with cosmological parameters $H_{0}=65 \mathrm{~km} \mathrm{~s}^{-1} \mathrm{Mpc}^{-1}, \Omega_{\Lambda}=0.7$, $\Omega_{M}=0.3$.

The continuous lines indicate the detection thresholds as functions of the redshift, for a typical GRB. Note that these are

${ }^{6}$ GRB 980425 has not been included in this calculation or in the successive ones because of its peculiarity.

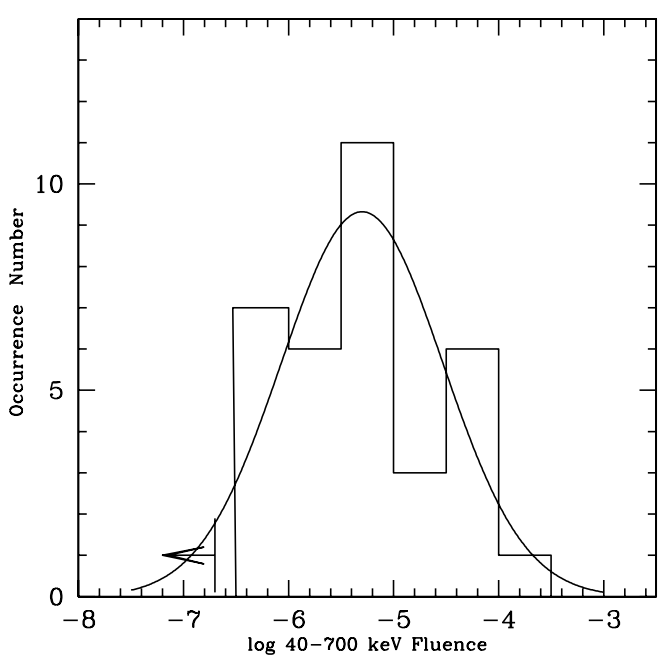

Fig. 4. The 40-700 keV fluence distribution of the BeppoSAX GRB sample. Data are extracted from the literature.

indicative values because the sensitivity depends on the off-axis angle and the event duration. The minimum energy required for a detection has been calculated taking the fluence detection thresholds of the two instruments to be around $S=10^{-7} \mathrm{erg} \mathrm{cm}^{-2}$ for the GRBM and $S=8 \times 10^{-8} \mathrm{erg} \mathrm{cm}^{-2}$ for the WFC. In the latter case, this corresponds to about $200 \mathrm{mCrab}$ in $20 \mathrm{~s}$. From the figures, it is evident that the gamma-ray energies are well above the GRBM threshold. On the contrary, the sample is limited by the WFC detection threshold, roughly corresponding to a isotropic energy in the $2-10 \mathrm{keV}$ range of $\sim 10^{50} \mathrm{erg}$ at $z=1$ and $\sim 10^{51} \mathrm{erg}$ at $z=4$.

We note, however, that this may not be true for X-ray rich GRBs and X-ray Flashes (Heise et al. 2002): the $\gamma$-ray emission of these objects is weak or absent. In these cases, only the WFC could detect distant events.

\subsection{Correlation between afterglow luminosity and gamma-ray energy}

We note that the width of the $\gamma$-ray fluence distribution is not very different from that of the X-ray afterglow flux distribution (see Figs. 2 and 4) A few authors, e.g. Kumar \& Piran (2000), 

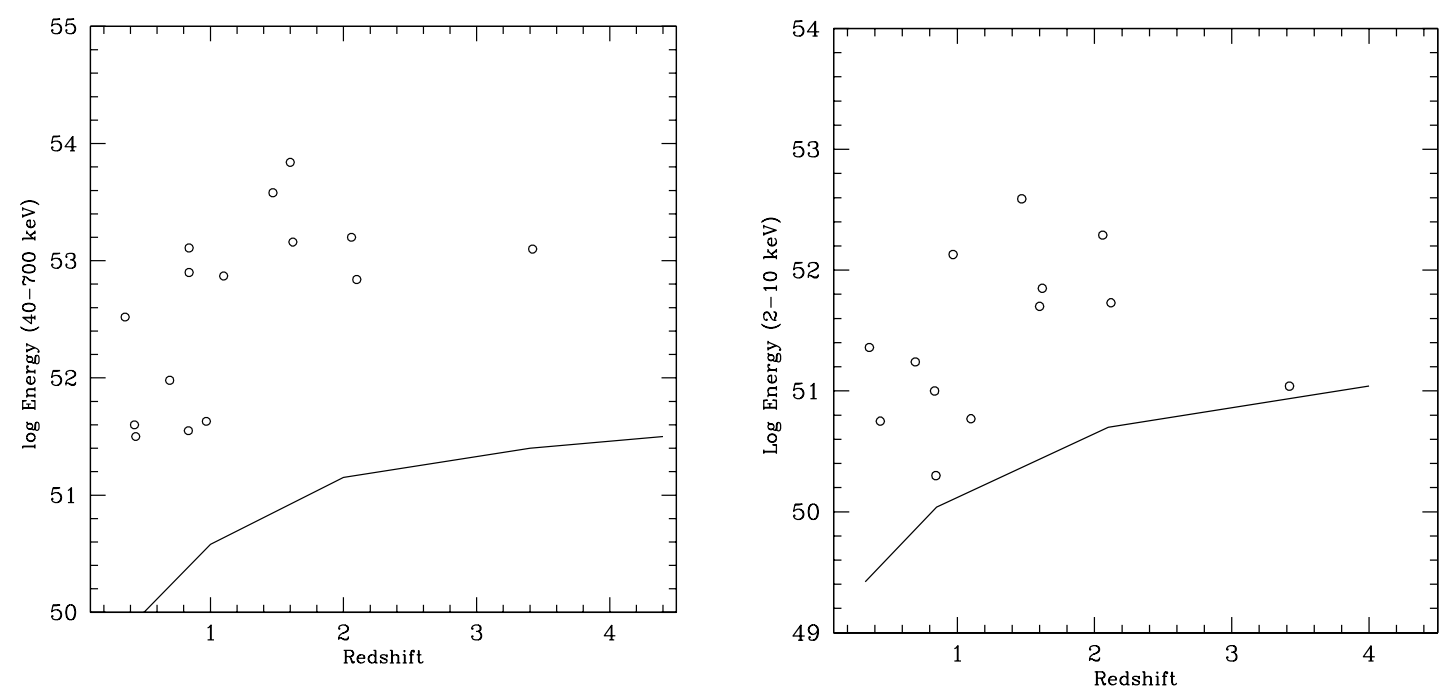

Fig. 5. Left: energy emitted during the prompt phase in the $40-700 \mathrm{keV}$ band in the burst rest frame. The solid line represents the detection threshold of the GRBM discussed in the text. Right: energy emitted during the prompt phase in the $2-10 \mathrm{keV}$ band in the burst rest frame. The solid line represents the detection threshold of the WFC discussed in the text.

have proposed that the energy emission from the fireball surface need not to be isotropic and that large spatial variations of $\mathrm{d} E_{\gamma} / \mathrm{d} \Omega$ in the fireball could exist. During the prompt emission phase, the radiation is highly beamed, due to the very high Lorentz factor of the ejecta. These circumstances would lead to a large spread of $\gamma$-ray fluences. In the afterglow phase, X-rays are beamed less, due to the lower Lorentz factor, and hence the fluctuations are averaged over a larger region. Therefore, X-ray flux afterglow distribution would be less broad than the $\gamma$-ray fluence. As we do not observe such a difference in the two distribution widths, we cannot support the hypothesis of Kumar \& Piran (2000).

The distribution of the $S_{\gamma} / F_{\mathrm{X}}$ ratio is not very broad ( $\sigma=$ 0.71 ), suggesting a correlation between the X-ray afterglow luminosity $L_{X}$ and the gamma-ray energy emitted $E_{\gamma}$ (see Fig. 6). Such a correlation may have important inferences on the fireball model. In fact, while the X-ray luminosity is a good indicator of the fireball blastwave energy, $E_{\gamma}$ is a natural measure of the radiated energy in the gamma-ray band. Therefore, the ratio between these quantities measures the $\gamma$-ray efficiency of these events. It should be noted that we compare $L_{\mathrm{X}}$ and $E_{\gamma}$ without any correction for a beaming effect: these quantities should be corrected by the same factor, so their ratio would not change.

For the sample of bursts with a known redshift, we derived $L_{X}$ by the formula (Lamb \& Reichart 2000):

$F(v, t)=\frac{L_{v}(v, t)}{4 \pi D^{2}(z)(1+z)^{1+\alpha-\delta}}$.

Luminosity is obtained in the $1.6-10 \mathrm{keV}$ energy band and at $11 \mathrm{~h}$ after the burst in the rest frame. We adopted the average values of $\alpha$ and $\delta$ reported in the previous section. The cosmological parameters used are the same as for the computation of the emitted energy (see Sect. 3.1) ${ }^{7}$.

In Fig. 7 we plot $L_{\mathrm{X}}$ vs. $E_{\gamma}$. The correlation coefficient is $r=0.74$, and the probability of chance correlation is 0.008 (Bevington \& Robinson 1992). It is worth noting that some indication of correlation between prompt and afterglow luminosity has already been found in a small set of Swift bursts (Chincarini et al. 2005), but our larger sample corroborates this result.

\footnotetext{
7 As for GRB 000214, $z=0.44$ was adopted.
}

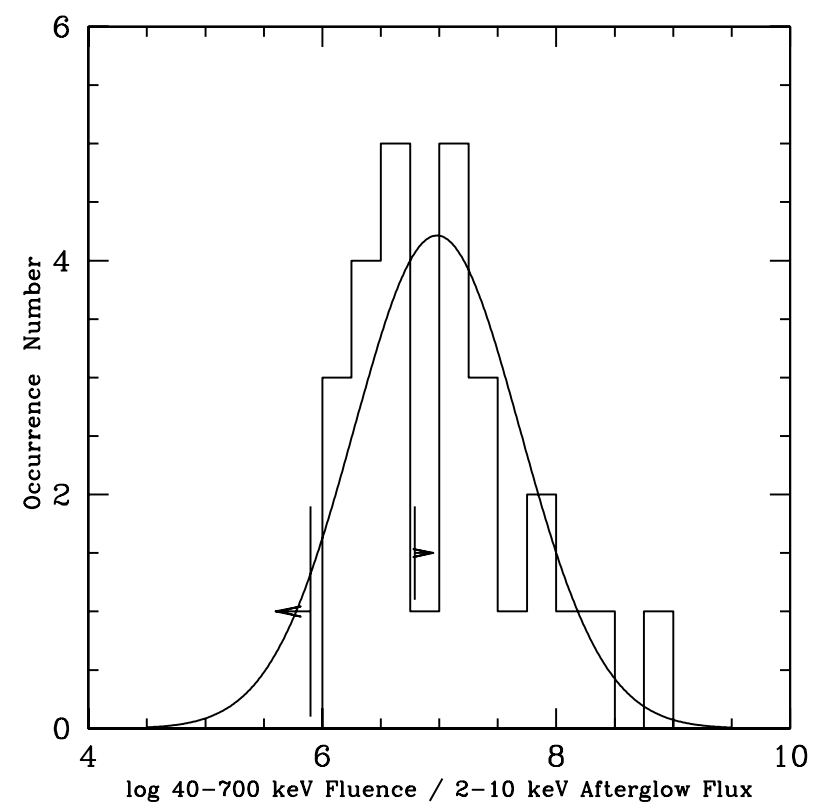

Fig. 6. Distribution of the logarithmic ratios of the prompt $\gamma$-ray fluence versus the X-ray afterglow flux for the BeppoSAX GRBsample.

Assuming that the observed X-ray frequency $v_{\mathrm{X}}$ is above the cooling frequency $v_{\mathrm{c}}$, the measurement of X-ray luminosity at a fixed time after the burst gives an estimate of isotropic kinetic energy of the fireball $E_{\mathrm{K}, \mathrm{A}}$ (Freedman \& Waxman 2001):

$E_{\mathrm{K}, \mathrm{A}}=C \epsilon_{\mathrm{e}}^{\frac{-4 p+4}{p+2}} v t L_{\mathrm{X}}$.

In that equation, $C$ is a parameter which depends very weakly on the fraction of energy carried by the magnetic field $\epsilon_{B}$, the luminosity distance, the flux density, the time $t$, and the frequency of observation $v$. $C$ has a stronger dependence on the value of $p$; however, we will make the simplifying assumption that the value of this parameter is the same for all bursts examined. For our purposes, the value of $C$ can thus be considered constant. We also note that Eq. (2) does not depend on the value of the density of the circumbust medium, so it holds either in the case of 


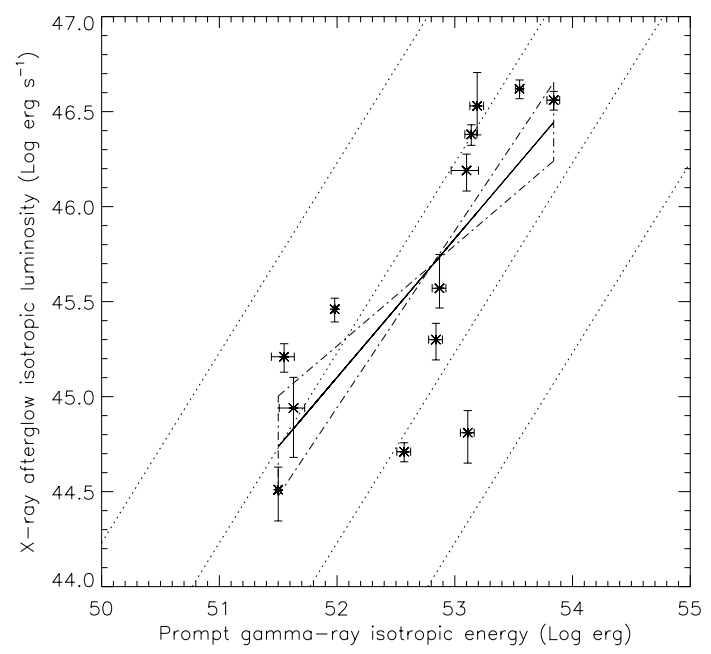

Fig. 7. 1.6-10 keV afterglow luminosity vs. 40-700 keV energy of the prompt emission. The fit between these two quantities, discussed in the text, is also shown, together with its confidence interval (dot-dashed box). The correlation coefficient is $r=0.74$. The dotted lines represent Eq. (5) when $\epsilon_{\mathrm{e}}^{1.3} / \epsilon_{\gamma}=10$ (upper line), 1 and 0.1 (middle lines), and 0.01 (lower line). The errors plotted here take the k-correction and measurement uncertainties into account.

expansion in the interstellar medium, with constant density, or in the case of a medium affected by the wind of the progenitor star, with a typical density profile decreasing as $r^{-2}$.

Using $p=2.4$, the value determined from the data, a luminosity distance of $3 \times 10^{28} \mathrm{~cm}$, a time and frequency of observation of $40000 \mathrm{~s}$ and $2.4 \times 10^{17} \mathrm{~Hz}$ respectively, and a flux density of $0.3 \mu \mathrm{Jy}, \epsilon_{B}=0.01$, Eq. (2) becomes:

$E_{\mathrm{K}, \mathrm{A}}=5.8 \times 10^{6} \epsilon_{\mathrm{e}}^{-1.3} L_{\mathrm{X}}$.

In the case of gamma-ray emission, we have to consider an unknown coefficient of conversion of relativistic energy of the fireball into gamma-ray energy (Piran et al. 2001).

$E_{\gamma}=\epsilon_{\gamma} E_{\mathrm{K}, \mathrm{P}}$

where $E_{\mathrm{K}, \mathrm{P}}$ is the isotropic relativistic energy of the fireball in the prompt phase. We may suppose $E_{\mathrm{K}, \mathrm{P}} \simeq E_{\mathrm{K}, \mathrm{A}}$ because $\epsilon_{\gamma}$ cannot be too close to unity, otherwise there will not be an afterglow (Kobayashi et al. 1997; Piran et al. 2001). We assume that radiative losses are also negligible. From the previous equations, we derive:

$L_{\mathrm{X}}=1.73 \times 10^{-7} \epsilon_{\mathrm{e}}^{1.3} \epsilon_{\gamma}^{-1} E_{\gamma}$.

We plot this relationship (dotted lines) in Fig. 7, assuming $\epsilon_{\mathrm{e}}^{1.3} / \epsilon_{\gamma}$ equal to $0.01,0.1,1$, and 10 , respectively. As one can see, the correlation we have found implies that the ratio $\epsilon_{\mathrm{e}}^{1.3} / \epsilon_{\gamma}$ does not strongly vary from burst to burst. Assuming that $\epsilon_{\mathrm{e}}$ is not too close to zero (a common value observed is $\sim 0.3$ Freedman \& Waxman 2001) - this implies that $\epsilon_{\mathrm{e}}$ is approximately proportional to $\epsilon_{\gamma}$. Thus, the fraction of fireball energy carried by relativistic electrons in the external shock and emitted in the afterglow is roughly proportional to the fraction of the fireball relativistic energy converted into $\gamma$-rays during the prompt phase.

\subsection{Jet collimation}

According to Sari et al. (1998), Chevalier \& Li (1999), and Rhoads (1997), the decay index and the spectral index values are

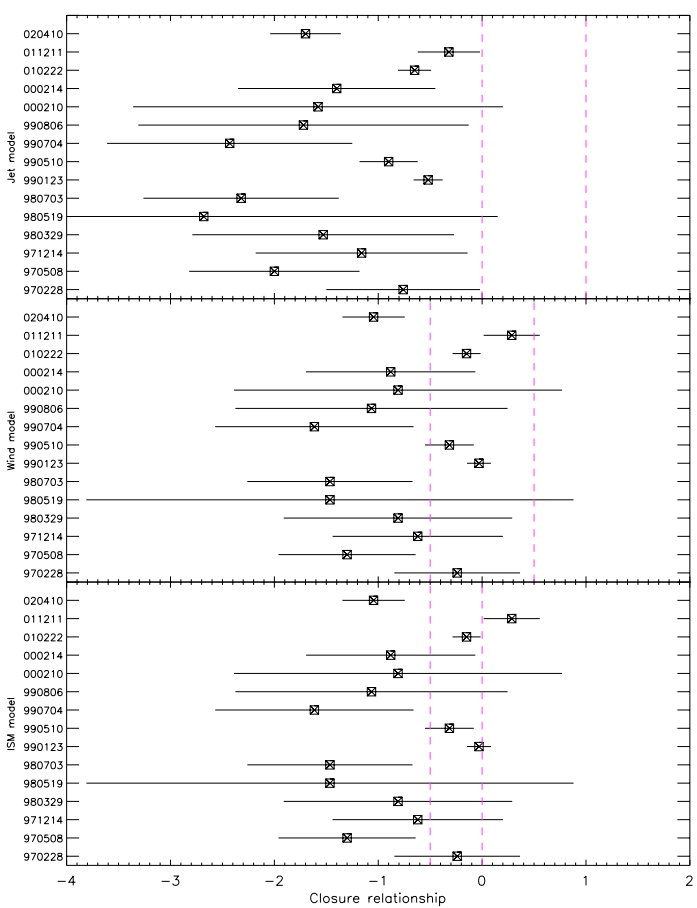

Fig. 8. The closure relationships for all burst with constraints on both the spectral and temporal decay indexes. We indicate the closure relationships for the three cases (Jet expansion, Wind model, ISM model) in the three panels. Vertical lines indicate the theoretical expected values.

linked together by closure relationships that depend on the burst geometry and environment. We present the closure relationships for each burst in Fig. 8, and focus first on the burst geometry (shown in the top panel of Fig. 8).

As one can see, in most of the cases the jet signature is ruled out by analysis. This is also evident when we calculate the mean value for the closure relationship. For a jet signature, this is:

$$
\begin{array}{rlr}
\delta-2 \alpha-1 & =-2.1 \pm 0.22 & v_{x}<v_{\mathrm{c}} \\
\delta-2 \alpha & =-1.1 \pm 0.22 & v_{x}>v_{\mathrm{c}} .
\end{array}
$$

In Eqs. (6) and (7), we should expect a value of 0, clearly excluded by the data. This implies that the beaming angle may be large. We can set a lower limit on its value $(\theta)$. According to Sari et al. (1999), we have:

$\theta=0.057\left(\frac{n_{-1}}{E_{\gamma, i, 53}}\right)^{1 / 8} t_{\theta, \text { day }}^{3 / 8}\left(\frac{\epsilon_{\gamma}}{0.2}\right)^{1 / 8}\left(\frac{1+z}{2}\right)^{-3 / 8}$

In Eq. (8), $E_{\gamma, i, 53}$ represents the isotropic energy emitted in $\gamma$-rays by the fireball in units of $10^{53} \mathrm{erg}, n_{-1}$ is the density in 0.1 particle $\mathrm{cm}^{-3}$ units, $\epsilon_{\gamma}$ is the efficiency of conversion of explosion energy into $\gamma$-rays, and $t_{\theta \text {,day }}$ is the date when the break of the light curve, due to the beamed emission, appears (expressed in days).

BeppoSAX TOOs are mostly carried out within 2 days after the GRB . Because decay and spectral slopes are not consistent with a collimated outflow, we can derive $t_{\theta \text {,day }}>2$. Assuming a typical $E_{\gamma, i, 53}=1, \epsilon_{\gamma}=0.2$, and $n_{-1}=100$ (Berger et al. 2003), we obtain a limit of $\theta \gtrsim 0.1 \mathrm{rad}$, which in turn give us a lower limit on the beaming factor $f_{\mathrm{b}} \approx 0.005$. This result is of the same order of magnitude of that claimed by Frail et al. (2001). We note that the majority of beaming angles, mostly inferred by breaks in optical light curves, are consistent with this result. Only GRB 990510 and GRB 010222 seem to represent 
Table 3. X-ray luminosity (assuming isotropy, $L_{\mathrm{X}}^{\text {iso }}$, and after beaming correction, $\left.L_{\mathrm{X}}^{\text {corr }}\right)$, energy emitted during the prompt $\gamma$-ray event (assuming isotropy, $E_{\gamma}^{\text {iso }}$ ) in units of $10^{51} \mathrm{erg}$, and beaming angle for BeppoSAX GRBs with a measured beaming angle (extracted from literature).

\begin{tabular}{ccccc}
\hline \hline GRB name & $\begin{array}{c}L_{\mathrm{X}}^{\text {iso }} \\
10^{44} \mathrm{erg} \mathrm{s}^{-1}\end{array}$ & $\begin{array}{c}E_{\gamma}^{\text {iso }} \\
10^{51} \mathrm{erg}\end{array}$ & $\begin{array}{c}\theta \\
\mathrm{rad}\end{array}$ & $\begin{array}{c}L_{\mathrm{X}}^{\text {corr }} \\
10^{44} \mathrm{erg} \mathrm{s}^{-1}\end{array}$ \\
\hline GRB 970228 & 28.6 & 9.9 & $>0.32$ & $>1.46$ \\
GRB 970508 & 16.1 & 3.5 & 0.391 & 1.23 \\
GRB 971214 & 147 & 125 & $>0.1$ & $>0.74$ \\
GRB 980613 & 7.21 & 4.26 & $>0.226$ & $>0.2$ \\
GRB 980703 & 37.4 & 74.1 & 0.2 & 0.75 \\
GRB 990123 & 373 & 692 & 0.089 & 1.48 \\
GRB 990510 & 269.7 & 144.5 & 0.054 & 0.39 \\
GRB 990705 & - & 79.4 & 0.096 & - \\
GRB 990712 & - & 3.32 & $>0.777$ & - \\
GRB 000210 & 6.96 & 130 & $>0.139$ & $>0.07$ \\
GRB 000214 & 3.4 & 3.17 & $>0.115$ & $>0.023$ \\
GRB 000926 & 335 & 155 & 0.140 & 2.14 \\
GRB 010222 & 377 & 375 & 0.08 & 13.1 \\
GRB 011121 & 5.1 & 3.74 & 0.145 & 0.05 \\
GRB 011211 & 20 & 68.8 & 0.115 & 0.12 \\
\hline
\end{tabular}

exceptions (see Table 3). A density of $n_{-1}=100$ is typical of the interstellar medium. On the other hand, the association between long GRBs and massive stars is proven with little doubt. GRB 980425 was a very peculiar burst. However, its association with SN1998bw linked massive stars and GRBs (e.g., Wang \& Wheeler 1998). Other spectroscopic associations between supernovae and GRBs, such as GRB 030329 and SN2003dh (Stanek et al. 2003; Hjorth et al. 2003) or GRB 031203 and SN20031w (Thomsen et al. 2004; Malesani et al. 2004), have proven this association. Zeh et al. (2004) have shown that in almost every case where we could have expected to see a SN bump, one was seen. Massive stars should produce the GRB within their original forming regions, which are usually very dense. If we assume $n_{-1}=10^{4}$, which is typical of giant molecular clouds, the beaming angle limit increase to $\theta \gtrsim 0.24 \mathrm{rad}$, which corresponds to a beaming factor limit of $f_{\mathrm{b}} \approx 0.03$.

Berger et al. (2003) claimed that the distribution of X-ray afterglow luminosity appears to converge significantly toward a common value after beaming correction. We have tested this hypothesis with our sample, using the beaming angle values reported in the literature (see Table 3; most of them are extracted from the article by Berger et al. 2003). The isotropic luminosity is corrected for beaming by applying a multiplicative factor depending on the beaming angle (see Berger et al. 2003, for details). Before beaming correction, the luminosity distribution displays a logarithmic width of 0.71 (see Fig. 9), with a mean value of $7.2 \times 10^{45} \mathrm{erg} \mathrm{s}^{-1}$. After the beaming correction, the distribution width is 0.55 . The mean luminosity decreases to $8 \times 10^{43} \mathrm{erg} \mathrm{s}^{-1}$ (Fig. 9). Thus, we do not have a clear indication of a shrinking of the luminosity distribution when taking beaming into account. We note that Gendre et al. (2005) find similar results on the basis of a set of X-ray afterglows observed by Chandra and XMM. One may note that the beaming angle was calculated, assuming a density of $10 \mathrm{~cm}^{-3}$ when it was unknown. This may have signifincant consequences. As an example, in't Zand et al. (2001) have reported a density value of $10^{6} \mathrm{~cm}^{-3}$ for GRB 010222. When using this value, rather than that reported by Berger et al. (2003), the beaming angle increases up to $0.26 \mathrm{rad}$. This leads the beaming-corrected luminosity distribution width to increase to a value of 0.7 , clearly no

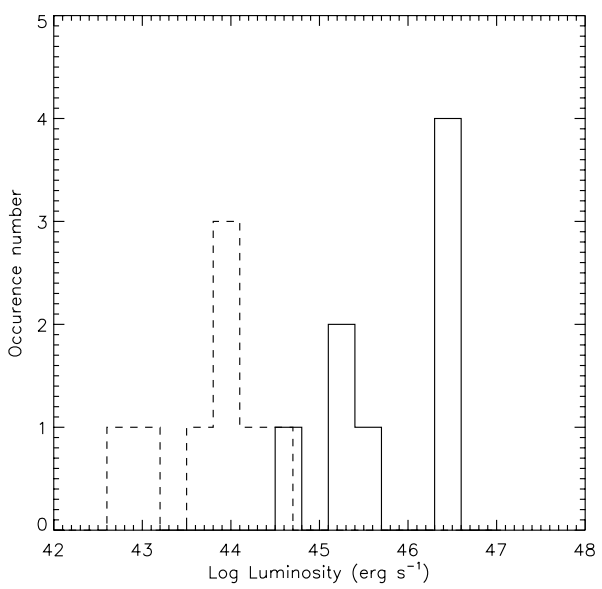

Fig. 9. Afterglow luminosity of BeppoSAX GRBs with known redshift. Solid line: before correction for beaming. Dashed line: after correction for beaming.

Table 4. Mean closure relationships from our sample. We indicate the wind and ISM closure relationships, depending on the cooling frequency position.

\begin{tabular}{ccc}
\hline \hline & ISM & Wind \\
\hline$v_{\mathrm{X}}<v_{\mathrm{c}}$ & $\delta-1.5 \alpha=-0.5 \pm 0.2$ & $\delta-1.5 \alpha-0.5=-1 \pm 0.2$ \\
$v_{\mathrm{c}}<v_{\mathrm{X}}$ & $\delta-1.5 \alpha+0.5=0 \pm 0.2$ & $\delta-1.5 \alpha+0.5=0 \pm 0.2$ \\
\hline
\end{tabular}

longer supporting the hypothesis of a standard energy release in the afterglow. Thus, such claims should be accepted with caution, depending on the assumptions made for the density values.

\subsection{The density profile of the environment}

Figure 8 also displays the closure relationships for an expansion into a wind environment (the WIND case, middle panel) and a constant density medium (the ISM case, bottom panel). These closure relationships present a degeneration when $v_{\mathrm{c}}<v_{\mathrm{X}}$, which prevents us from drawing any conclusions. One can see from Fig. 8 that most of the bursts are in that situation. The uncertainties of other bursts do not allow us to draw any conclusions for most of them, using only the X-ray data. This is also shown by the mean closure relationships reported in Table 4: the two medium cases can fit the mean value if the cooling frequency is below the X-rays, while none of them can fit the mean value in the opposite case.

To get rid of this degeneration, we also need to use the optical observations. From the fireball model, the X-ray decay index is larger than the optical one, if the cooling frequency is between the optical and X-ray bands and if the fireball is expanding into a constant density medium (Sari et al. 1998). The difference between the optical and X-ray decay index is -0.25 . If the fireball expands into a wind environment (also assuming the cooling frequency to be between the optical and X-ray bands), then it is the optical decay index which is larger than the X-ray decay index. The difference between the optical and X-ray decay index is now 0.25 . Assuming that the cooling frequency is indeed between the optical and the X-ray bands, we can remove the degeneration.

In Table 5, we show the optical vs. X-ray band decay indexes (results taken from the literature). We excluded GRB 980519 and GRB 000926 from our set because in their case the jet phase started slightly after the beginning of BeppoSAX observations 
Table 5. Optical decay indexes and comparison with the X-ray band decay indexes.

\begin{tabular}{cccc}
\hline \hline GRB & $\delta_{\mathrm{O}}$ & $\delta_{\mathrm{X}}-\delta_{\mathrm{O}}$ & Reference \\
\hline GRB 970228 & $1.21 \pm 0.02$ & $0.1 \pm 0.2$ & 1 \\
GRB 970508 & $0.15 \pm 0.02$ & $0.65 \pm 0.2$ & 2 \\
GRB 971214 & $1.20 \pm 0.02$ & $-0.2 \pm 0.3$ & 3 \\
GRB 980329 & $1.3 \pm 0.2$ & $0.1 \pm 0.6$ & 4 \\
GRB 980613 & $0.8 \pm 0.5$ & $0.7_{-2.0}^{+1.1}$ & 5 \\
GRB 980703 & $1.2 \pm 0.4$ & $-0.1 \pm 0.5$ & 6 \\
GRB 990123 & $1.1 \pm 0.4$ & $0.3 \pm 0.4$ & 7 \\
GRB 990510 & $0.8 \pm 0.2$ & $0.6 \pm 0.3$ & 8 \\
GRB 010222 & $1.32 \pm 0.03$ & $0.03 \pm 0.07$ & 9 \\
GRB 011121 & $1.6 \pm 0.6$ & $-0.3 \pm 0.6$ & 10 \\
GRB 011211 & $0.95 \pm 0.2$ & $1.2 \pm 0.4$ & 11 \\
GRB 020322 & $0.5 \pm 0.3$ & $0.3 \pm 0.5$ & 12,13 \\
\hline
\end{tabular}

References: 1: Masetti et al. (1998), 2: Galama et al. (1998, the index shown is relative to the BeppoSAX observation interval), 3: Diercks et al. (1998), 4: Reichart et al. (1999), 5: Hjorth et al. (2002), 6: Bloom et al. (1998), 7: Kulkarni et al. (1999), 8: Harrison et al. (1999), 9: Masetti et al. (2001), 10: Price et al. (2002), 11: Jacobsson et al. (2003), 12: Bloom et al. (2002), 13: Greiner et al. (2002).

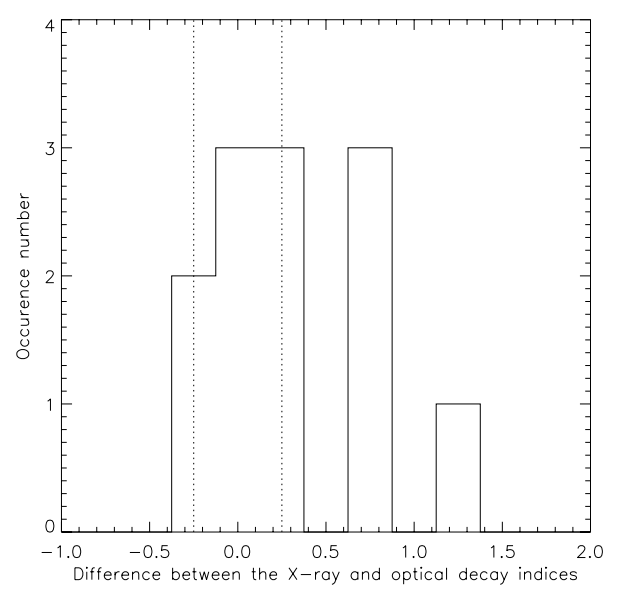

Fig. 10. Difference of the X-ray and optical decay indexes of BeppoSAX sample. Right line: $\delta_{\mathrm{X}}=\delta \mathrm{o}+0.25$ (as expected for an ISM environment). Left line: $\delta_{\mathrm{X}}=\delta \mathrm{o}-0.25$ (as expected for a wind environment). Uncertainties on the decay index values were not taken into account when building the histogram.

(Jaunsen et al. 2001; Fynbo et al. 2001); therefore, their decaying behavior may be largely affected by the change of slope.

For the remaining GRBs with both X-ray and optical afterglows detected, the average value of the decay index is $\delta_{\mathrm{O}}=$ $1 \pm 0.2$ in the optical and $\delta_{\mathrm{X}}=1.3 \pm 0.2$ in the X-rays. The difference between these two values is $0.3 \pm 0.3$. A constant density medium surrounding the burst is thus favored, but a wind environment is not ruled out. This is also visible in Fig. 10, where we plot the $\delta_{\mathrm{X}}-\delta_{\mathrm{O}}$ value for each single burst. For a majority of them, the value 0.25 is preferred, thus implying that we observe a constant density medium surrounding the burst, while for some others, we observe a wind medium. One should expect the surrounding medium to be the wind arising from the star for all bursts (Chevalier \& Li 1999). Ramirez-Ruiz et al. (2001) suggested the existence of a termination shock that could maintain the wind close to the star (see also Chevalier et al. 2004). This would explain our results. In such a case, this implies that the termination shock has been crossed before the observations (therefore early after the burst), which should then imply a dense surrounding medium. This is supported by the large absorption observed around the bursts (see Table 1): such a high density column may be due to a compact and dense layer around the burst. This is also supported by the observation of GRB 010222 . For this burst, the surrounding medium is indeed the interstellar medium (see Fig. 8); in't Zand et al. (2001) have proposed that this burst is surrounded by a very dense $\left(10^{6} \mathrm{~cm}^{-3}\right)$ medium or affected by a jet effect. We can discard the hypothesis of a jet effect (see Fig. 8), and thus confirm the proposed explanation of a very dense medium. Such a medium, with a large density, would be very efficient for maintaining the termination shock near the GRB progenitor.

Finally, we would like to underline the fact that inferences drawn from our afterglow analysis are in general agreement with those of the reviews of Frontera et al. (2003) and Piro (2004). This is not very surprising, however, because our results and those of Frontera et al. (2003) were consistent one another, and Piro (2004) used a large part of the same GRB X-ray afterglow set and basically the same data analysis to derive his conclusions.

\section{Dark GRBs}

BeppoSAX detected an X-ray afterglow in 31 of 36 followup observations of GRBs. This represents $86 \%$ of the sample. In 3 other cases, a single source was found, with little probability that it was a serendipitous one. Only in 2 cases were no $\mathrm{X}$-ray sources detected in the error circle, whereas only 16 GRBs present an optical afterglow. Taking into account the late followup of GRB 960720 and the absence of optical observations of GRB 980515 and GRB 020427, this implies that only $42 \%$ of the GRBs detected by BeppoSAX have an identified optical afterglow. The remaining part, with a detected X-ray afterglow, but no optical emission discovered, were called dark bursts. Several authors (e.g., Fynbo et al. 2001; Berger et al. 2002; Fox et al. 2003; Rol et al. 2005) pointed out that this definition can in fact hide an instrumental bias, as it holds irrespective of the date of the observation, the decay rate, the observed limiting magnitude at that date, and the expected brightness of the optical afterglow. In fact, the non-detection of the optical afterglow can be due to several reasons: a late follow-up, a steep decay, an intrinsic faintness, a large dust extinction, or a distant burst. While the first two possibilities are instrumental bias, the last three give information about the burst.

Other methods have been proposed to classify a burst as dark (e.g., Jakobsson et al. 2004), but they are based on a chromatic effect and do not enable us to assess if the afterglow was simply a faint source. Thus, in this paper, we shall define "dark bursts" the GRBs for which fast optical follow-up observations (the ones which took place less than $\sim 1$ day after the burst), at least as deep as $R_{\text {lim }}>19.5$, did not lead to a detection of an afterglow. For these objects, it has been shown that on average the optical flux should be 2 mag lower than for bursts with an optical afterglow in order to explain the non-detection of the optical source (Lazzati et al. 2002). Another study made with a sample of 31 BeppoSAX GRB afterglows indicated that the X-ray afterglow fluxes of dark GRBs are, on average, 4.8 times weaker than those of normal bursts (De Pasquale et al. 2003). The probability that this flux distribution comes from a single population of bursts is 0.002 , i.e., a $3 \sigma$ rejection. Using the whole BeppoSAX sample, this probability does not change significantly.

The results exposed in Sect. 3.3 imply that this X-ray faintness should extend to the prompt phase, and thus that dark GRBs should present a fainter $\gamma$-ray fluence. We have tested this hypothesis and present the results in Fig. 11. As one can see, there is indeed a trend for the dark burst (dotted line) to 


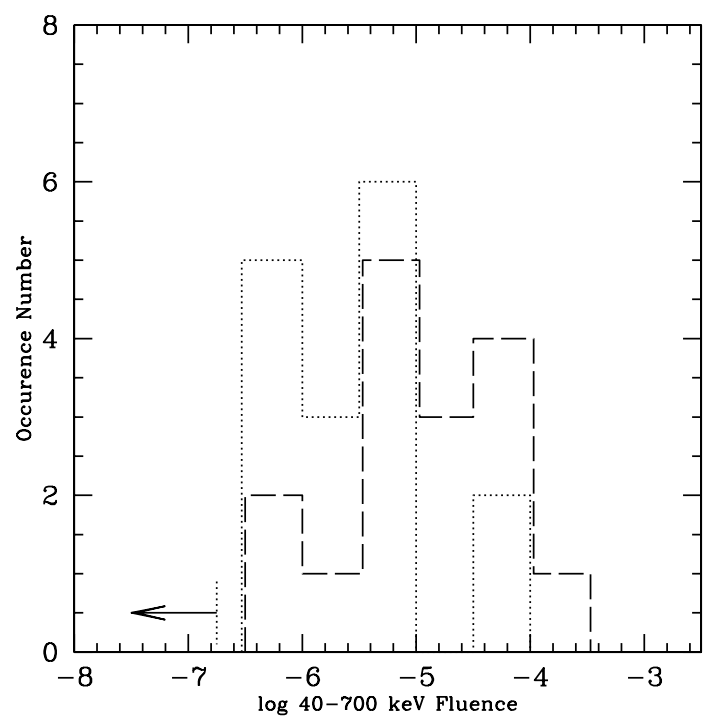

Fig. 11. Comparison of the $\gamma$-ray fluences of dark (dotted line) and optically bright (dashed line) GRBs.

have a low $\gamma$-ray fluence, compared to GRBs with optical transient (OT GRBs). The ratio between the average dark GRB fluence and OT GRBs fluence is 5.7, similar to the value of the ratio of X-ray fluxes and the expected value derived from the correlation observed in Sect. 3.3. The probability that optically bright GRBs, and dark GRBs, fluence distributions derive from a unique population of burst is 0.01 . It thus seems that faintness is an intrinsic property of dark GRBs at all wavelengths.

The above statements can explain the non-detection of the optical afterglow. But they imply that the whole afterglow is affected by this effect (i.e., the faintness is observed in all the observation bands). On the contrary, extinct optical afterglow and distant bursts should also feature a faintness that is wavelength dependent (due to dust-to-gas laws in the first case and due to the Lymann- $\alpha$ forest redshifted in the optical band in the second case). To discriminate among all these effects and to validate their interpretation, De Pasquale et al. (2003) also carried out a comparison of the X-ray and optical fluxes. They found that $75 \%$ of dark bursts were compatible with a global faintness, and thus that these bursts were dark because searches were not fast or deep enough.

For the remaining GRBs, the optical-to-X-ray flux ratio is at least a factor of 5-10 lower than the average value observed in normal GRBs. In terms of the spectral index, these events have optical-to-X-ray spectral indexes of $\alpha_{\mathrm{OX}} \lesssim 0.6$, whereas for OT GRBs the average value is $\simeq 0.75$. These facts strongly suggest that for these bursts, the spectrum is depleted in the optical band. Jacobsson et al. (2004), using a similar method and comparing their results with the fireball model expectations, indicated that at least $10 \%$ of their sample was not compatible with the fireball model and thus were truly dark GRBs. It is worth noting that the recently begun Swift mission (Gehrels et al. 2005) has already confirmed that a considerable fraction of GRBs has tight upper limits for the optical emission (Roming et al. 2005, in preparation) We can thus indicate that about 10-20\% of GRBs are characterized by an optical afterglow emission fainter than that expected from the X-ray afterglow flux. These bursts could be distant $(z>5)$ or extinct bursts.

Two dark bursts have been associated with host galaxies at $z<5$ (Djorgovski et al. 2001; Piro et al. 2002). We also note (see Table 1) that the X-ray absorption around some bursts is important and could be responsible for an important optical extinction (see, e.g., Stratta et al. 2004). Thus, for some of these events, the likely explanation of the darkness is an optical depletion by dust in the star-forming region. This in turn supports the massive star progenitor hypothesis for long GRBs, as these massive stars are likely to explode in their original star-forming region. On the other hand, this does not rule out the distance explanation for some dark bursts with no known host. In fact, it is likely that the dark burst population is the sum of these three (faint, distant, and extinct) populations. In principle, these cases could be disentangled by other measurements, such as column density, prompt $E_{\text {peak }}$, or X-ray flux. However, it is important to be cautious because a few X-ray flashes (see Heise et al. 2001) could have the values of these parameters consistent with those of very high redshift GRBs, even if they are not actually placed at $z>5$.

\section{Conclusions}

We have presented the BeppoSAX X-ray afterglow catalog. Thirty-nine BeppoSAX afterglow observations were carried out on a sample of 52 detected GRBs. Thirty-one X-ray afterglows were securely identified due to their fading behavior. Three other observations led to the detection of only one source within the prompt positional error box. Thus, X-ray afterglows are present in $\sim 90 \%$ of the observations.

We derived the main properties - flux, decay index, spectral index, and absorption - for 15 afterglows and give constraints on decay slope and flux for the remaining ones. The width of the prompt fluence and X-ray afterglow flux distributions are similar, suggesting no strong spatial variation of the energy emission within the beamed fireball. We pointed out a likely correlation between the X-ray afterglow luminosity and the energy emitted during the prompt $\gamma$-ray event. Such a correlation suggests that the fraction of fireball energy carried by relativistic electrons in the external shock and emitted in the afterglow is roughly proportional to the fraction of the fireball relativistic energy converted into $\gamma$-ray during the prompt phase.

We do not detect a significant jet signature within the afterglow observations, implying a lower limit on the beaming angle of $\sim 0.1$. Moreover, we note that the hypothesis of a standard energy release in the afterglow, as claimed by Berger et al. (2003), may be consistent with our sample, but it strongly depends on the assumptions made about the density of the surrounding medium.

The average value of the spectral index of the electron energy distribution, inferred by our time and spectral analysis, is $p=2.4 \pm 0.2$.

Our data support the fact that GRBs should typically be surrounded by a medium with a constant density rather than a wind environment and that this medium should be dense. This may be explained by a termination shock located near the burst progenitor. We finally pointed out that some bursts without optical counterparts may be explained by an intrinsic faintness of the event, while others can be strongly absorbed.

A first comparison with the bursts observed by XMM-Newton and Chandra are presented in Gendre et al. (2006). In a forthcoming paper (Gendre et al., in preparation), we will search the spectra for metal lines and other deviations from the continuum properties.

Acknowledgements. The BeppoSAX satellite was a joint program of Italian (ASI) and Dutch (NIVR) space agencies. BG acknowledges support by the EU FP5 RTN "Gamma ray bursts: an enigma and a tool". We would like to thank the referee for his comments. 


\section{References}

Amati, L. 1999, private comunication

Amati, L., Frontera, F., Costa, E., \& Feroci, M. 1998, GCN \#146

Amati, L., Frontera, F., Tavani, M., et al. 2002, A\&A, 390, 81

Amati, L., Frontera, F., Castro-Ceron, J. M., et al. 2003, Prooceedings of GRB and Afterglow Astronomy 2001, AIP Conf. Proc., 662, 387

Amati, L., Frontera, F., in't Zand, J., et al. 2004, A\&A, 426, 415

Antonelli, L. A., Fiore, F., Amati, L., et al. 1999, A\&AS, 138, 435

Antonelli, L. A., Piro, L., Vietri, M., et al. 2000, ApJ, 545L, 39

Berger, E., Kulkarni, S. R., Bloom, J. S., et al. 2002, ApJ, 581, 981

Berger, E., Kulkarni, S. R., \& Frail, D. A. 2003, ApJ, 590, 379

Bevington, F. R., \& Robinson, D. K. 1992, Data Reduction and Error Analysis for the Physical Sciences (2nd edition, McGraw Hill)

Bloom, J. S., Frail, D. A., Kulkarni, S. R., et al. 1998, ApJ, 508, L21

Bloom, J. S., Mirabal, N., Helpern, J. P., Fox, D. W., \& Lopes, P. A. A. 2002 GCN \#1296

Boella, G., Butler, R. C., Perola, G. C., et al. 1997, A\&AS, 122, 299

Borozdin, K. N., \& Trudolyubov, S. P. 2003, ApJ, 583, 57

Chevalier, R. A., \& Li, Z. Y. 1999, ApJ, 520, L29

Chevalier, R. A., Li, Z. Y., \& Fransson, C. 2004, ApJ, 606, 369

Chincarini, G., Moretti, A., Romano, P., et al. 2005, ApJ, submitted [arXiv: astro-ph/0506453]

Cornelisse, R., Verbunt, F, in 't Zand, J., et al. 2002, A\&A, 392, 885

Costa, E., Frontera, F., Heise, J., et al. 1997, Nature, 387, 783

Dal Fiume, D., Amati, L., \& Antonelli, L. A. 2000, A\&A, 355, 454

De Pasquale, M., Piro, L., Perna, R., et al. 2003, ApJ, 592, 1018

Dickey, J. M., \& Lockman, F. J. 1990, ARA\&A, 28, 215

Diercks, A. H., Deutsch, E. W., Castander, F. J., et al. 1998, ApJ, 503, L105

Djorgovski, S. G., Frail, D. A., Kulkarni, S. R., et al. 2001, ApJ, 562, 654

Feroci, M., Antonelli, L. A., Guainazzi, M., et al. 1998, A\&A, 332, L29

Feroci, M., Antonelli, L. A., Soffitta, P., et al. 2001, A\&A, 378, 441

Fiore, F., Guinazzi, M., \& Grandi, P. 1999, Handbook for BeppoSAX NFI Spectral Analysis,

ftp:www.sdc.asi.it/pub/sax/doc/software_docs/ saxabc_v1.2.ps.gz

Fox, D. W., Price, P. A., Soderberg, A. M., et al. 2003, ApJ, 586, L5

Frail, D. A., Kulkarni, S. R., Nicastro, S. R., Feroci, M., \& Taylor, G. B. 1997, Nature, 389, 261

Frail, D. A., Kulkarni, S. R., Sari, R., et al. ApJ, 2001, 562, L55

Frontera, F. 2003, Lect. Notes Phys., 598, 317

Frontera, F. 2004, Proceedings of GRBs in the afterglow Era 2002, ASP Conf. Ser., 312,3

Freedman, D., \& Waxman, E. 2001, ApJ, 547, 922

Frontera, F., Costa, E., Dal Fiume, D., et al. 1997, A\&AS, 122, 357

Frontera, F., Costa, E., Dal Fiume, D., et al. 1998, ApJ, 493L, 67

Frontera, F., Amati, L., Costa, E., et al. 2000a, ApJS, 127, 59

Frontera, F., Antonelli, L. A., Amati, L., et al. 2000b, ApJ, 540, 697

Fynbo, J. U., Jensen, B. L., Gorosabel, J., et al. 2001, A\&A, 369, 373

Galama, T. J., Groot, P. J., van Paradijis, J., et al. 1998, ApJ, 497, L13

Gandolfi, G., Soffitta, P., Heise, J., et al. 1999, GCN \#448

Gehrels, N., Chincarini, G., Giommi, P., et al. 2005, ApJ, 611, 1005

Gendre, B., Corsi, A., \& Piro, L. 2006, A\&A, 455, 803

Giommi, P., Perri, M., \& Fiore, F. 2000, A\&A, 362, 799

Greiner, J., Thiele, U., Klose, S., \& Castro-Tirado, A. J. 2002, GCN \#1298

Guidorzi, C., Montanari, E., Frontera, F., et al. 2000, GCN \#675

Guidorzi, C., Frontera, F., Montanari, E., et al. 2003, A\&A, 401, 491

Harrison, F. A., Bloom, J. S., Frail, D. A., et al. 1999, ApJ, 523, L121

Heise, J., in 't Zand, J., Kippen, M., et al. 2002, Proceedings of the 2000 Rome

Workshop on Gamma Ray Burst in the Afterglow Era, AIP, 229

Hjorth, J., Thomsen, B., Nielsen, S. R., et al. 2002, ApJ, 576, 113

Hjorth, J., Sollerman, J., Møller, P., et al. 2003, Nature, 423, 847

Hurley, K., Mazets, E., Golenetskii, S., et al. 2000, GCN 801

Jakobsson, P., Hjorth, J., Fynbo, J. P. U., et al. 2003, A\&A, 408, 941

Jakobsson, P., Hjorth, J., Fynbo, J. P. U., et al. 2004, ApJ, 617, L21

Jager, R., Mels, W. A., Brinkman, A. C., et al. 1997, A\&AS, 125, 557

Jaunsen, A. O., Hjorth, J., Björnsson, G., et al. 2001, ApJ, 546, 127

Klebesadel, R. W., Strong, I. B., \& Olson, R. A. 1973, ApJ, 182, L85

Kobayashi, S., Piran, T., \& Sari, R. 1997, ApJ, 490, 92
Kulkarni, S. R., Djorgovski, S. G., Odewahn, S. C., et al. 1999, Nature, 398, 389 Kuulkers, E., Antonelli, L. A., Kuiper, L., et al. 2000, ApJ, 538, 638 Kumar, P., \& Piran, T. 2000, ApJ, 535, 152

Lamb, D., \& Reichart, E. 2000, ApJ, 536, 1

Lazzati, D., Covino, S., \& Ghisellini, G. 2002, MNRAS, 330, 583

Levine, A., Morgan, E. \& Muno, M. 1998, IAUC 6966

Maccacaro, T., Gioia, I. M., Wolter, A., et al. 1988, ApJ, 326, 680

Maiorano, E., Masetti, N., Palazzi, E., et al. 2005, A\&A, 438, 821

Malesani, D., Tagliaferri, G., Chincarini, G., et al. 2004, ApJ, 609, L5

Masetti, N., Bartolini, C., Guarnieri, A., \& Piccioni, A. 1998, Proceedings of the Active X-ray Sky symposium 1997, ed. L. Scarsi, H. Bradt, P. Giommi, \& F. Fiore, 674

Masetti, N., Palazzi, E., Pian, E., et al. 2001, A\&A, 374, 382

Meszaros, P., \& Rees, M. J. 1997, ApJ, 476, 232

Metzger, M. R., Djorgovski, S. G., Kulkarni, S. R., et al. 1997, Nature, 387, 879

Montanari, E., Amati, L., Frontera, F., et al. 2002, Proceedings of 2nd Rome Workshop on Gamma-Ray Burst in the afterglow Era, 195

Muller, J. M., Costa, E., Gandolfi, G., et al. 1999b, IAUC 7211

Nicastro, L., Amati, L., Antonelli, L. A., et al. 1998, A\&A, 338, L17

Nicastro, L., Amati, L., Antonelli, L. A., et al. 1999, A\&AS, 138, 437

Nicastro, L., Cusumano, G., Antonelli, L. A., et al. 2001, Proceedings of GRBs in the afterglow Era 2000, ed. E. Costa, F. Frontera, \& J. Hjorth, 198

Nicastro, L., in't Zand, J., Amati, L., et al. 2004, A\&A, 427, 445

Panaitescu, A., Meszaros, P., \& Rees, M. J. 1998, ApJ, 503, 314

Panaitescu, A., \& Kumar, P. 2002, ApJ, 571, 779

Parmar, A. N., Martin, D. D. E., Bavdaz, M., et al. 1997, A\&AS, 122, 309

Pian, E., Amati, L., Antonelli, L. A., et al. 1999, A\&AS, 138, 463

Pian, E., Amati, L., Antonelli, L. A., et al. 2000, ApJ, 536, 778

Piran, T., Kumar, P., Panaitescu, A., \& Piro, L. 2001, ApJ, 560, L167

Piro, L. 1995, SAX Observer Handbook, Agenzia Spaziale Italiana, Issue 1.0, ed. L. Piro

Piro, L. 2004, Proceedings of GRBs in the afterglow Era 2002, ASP Conf. Ser., 149

Piro, L., Heise, J., Jager, R., et al. 1998a, A\&A, 329, 906

Piro, L., Amati, L., Antonelli, L. A., et al. 1998b, A\&A, 331, L41

Piro, L., Costa, E., Feroci, M., et al. 1999, 514, L73

Piro, L., Garmire, G., Garcia, M., et al. 2001, ApJ, 558, 442

Piro, L., Frail, D. A., Gorosabel, J., et al. 2002, ApJ, 577, 680

Piro, L., De Pasquale, M., Soffita, P., et al. 2005, ApJ, 623, 314

Price, P., Berger, E., Reichart, D. E., et al. 2002, ApJ, 572, L51

Ramirez-Ruiz, E., Dray, L. M., Madau, P., \& Tout, C. A. 2001, MNRAS, 327, 829

Rees, M. J., \& Meszaros, P. 1992, MNRAS, 258, 41

Reeves, J. N., Watson, D., Osborne, J. P., et al. 2002, Nature, 416, 512

Reichart, D. E., Lamb, D. Q., Metzger, M. R., et al. 1999, ApJ, 517, 692

Rhoads, J. E. 1997, ApJ, 487, L1

Rol, E., Wijers, R. A. M. J., Kouveliotou, C., Kaper, L., \& Kaneko, Y. 2005, ApJ, 624,868

Sari, R., Piran, T., \& Narayan, N. 1998, ApJ, 497, L17

Sari, R., Piran, T., \& Helpern, J. P. 1999, ApJ, 519, L17

Soffitta, P., Amati, L., Antonelli, L. A., et al. 2002, Proceedings of GRB in the Afterglow Era 2000, 201

Stanek, K. Z., Matheson, T., Garnavich, P. M., et al. 2003, ApJ, 591, L17

Stratta, G., Fiore, F., Antonelli, L. A., et al. 2004, ApJ, 608, 846

Tassone, G., in 't Zand, J., Frontera, F., \& Gandolfi, G. 1999, IAUC \#7281

Thomsen, B., Hjorth, J., Watson, D., et al. 2004, A\& A, 419, L21

van Paradijs, J., Groot, P. J., Galama, T. J., et al. 1997, Nature, 386, 686

Vreeswijk, P. M., Galama, T. J., \& Owens, A. 1999, ApJ, 528, 171

Wang, L., \& Wheeler, J. C. 1998, ApJ, 504, L87

Woosley, S. 1993, ApJ, 405, 273

in 't Zand, J., Amati, L., Antonelli, L. A., et al. 1998, ApJ, 505, L119

in't Zand, J., Heise, J., van Paradijs, J., \& Fenimore, E. E. 1999, ApJ, 516, L57

in't Zand, J., Heise, J., Kuulkers, E., et al. 2000a, GCN \#677

in't Zand, J., Kuiper, L., Amati, L., et al. 2000b, ApJ, 545, 266

in't Zand, J., Kuiper, L., Amati, L., et al. 2001, ApJ, 559, 710

in't Zand, J., Kuiper, L., Heise, J., et al. 2004, Proceedings of GRBs in the afterglow Era 2002, ASP Conf. Ser., 209

Zeh, A., Klose, S., \& Hartmann, D. H. 2004, ApJ, 609, 952 
M. De Pasquale et al.: BeppoSAX GRB X-ray afterglows catalog, Online Material $p 1$

\section{Online Material}


M. De Pasquale et al.: BeppoSAX GRB X-ray afterglows catalog, Online Material p 2

Table 6. GRBs localized and/or observed by BeppoSAX. We indicate the position of the first TOO start and end times, the sum of the Good Time Interval (GTI), and the date of the subsequent TOOs. "WFC" following the position means that this GRB was localized only the the WFC, "NFI" a localization obtained by NFI. An external trigger of a BeppoSAX TOO is indicated by giving the satellite that localized the burst in parenthesis, but the localization displayed has been provided by NFI. We also indicate in the table if an optical afterglow was detected together with the distance and other information obtained from the optical study.

\begin{tabular}{|c|c|c|c|c|c|c|c|}
\hline GRB name & $\begin{array}{r}\mathrm{Po} \\
\text { (right } \\
\text { decl }\end{array}$ & $\begin{array}{l}\text { ition } \\
\text { cension, } \\
\text { ation) }\end{array}$ & Localization & $\begin{array}{l}\text { First TOO } \\
\text { start-end } \\
\text { (h) }\end{array}$ & $\begin{array}{c}\text { Sum of } \\
\mathrm{GTI}^{a} \\
(\mathrm{ks})\end{array}$ & $\begin{array}{l}\text { Other TOOs } \\
\text { start-end } \\
\text { (h) }\end{array}$ & $\begin{array}{l}\text { Optical afterglow } \\
\text { detection (redshift) }\end{array}$ \\
\hline GRB 960720 & $17^{\mathrm{h}} 30^{\mathrm{m}} 37^{\mathrm{s}}$ & $+49^{\circ} 05^{\prime} 48^{\prime \prime}$ & $\overline{\text { WFC }}$ & $3715-3765.2$ & 49.1 & - & $\mathrm{N}$ \\
\hline GRB 970111 & $15^{\mathrm{h}} 28^{\mathrm{m}} 10^{\mathrm{s}}$ & $+19^{\circ} 36^{\prime} 17^{\prime \prime}$ & NFI & $16-46.5$ & 56 & - & $\mathrm{N}$ \\
\hline GRB 970228 & $05^{\mathrm{h}} 01^{\mathrm{m}} 47^{\mathrm{s}}$ & $+11^{\circ} 46^{\prime} 41^{\prime \prime}$ & NFI & $8-16.7$ & 14.3 & $89.6-98.8$ & $Y(z=0.695)$ \\
\hline GRB 970402 & $14^{\mathrm{h}} 50^{\mathrm{m}} 03^{\mathrm{s}}$ & $-69^{\circ} 20^{\prime} 06^{\prime \prime}$ & NFI & $8-19$ & 23.6 & $40.9-58.5$ & $\mathrm{~N}$ \\
\hline GRB 970508 & $06^{\mathrm{h}} 53^{\mathrm{m}} 49^{\mathrm{s}}$ & $+79^{\circ} 16^{\prime} 20^{\prime \prime}$ & NFI & $6-21.6$ & 35.5 & $\begin{array}{c}66-74 \\
136.3-160\end{array}$ & $\mathrm{Y}(z=0.835)$ \\
\hline GRB 971214 & $11^{\mathrm{h}} 56^{\mathrm{m}} 25^{\mathrm{s}}$ & $+65^{\circ} 12^{\prime} 43^{\prime \prime}$ & NFI & $6.5-60.7$ & 101 & - & $\mathrm{Y}(z=3.42)$ \\
\hline GRB 971227 & $12^{\mathrm{h}} 57^{\mathrm{m}} 15^{\mathrm{s}}$ & $+59^{\circ} 23^{\prime} 26^{\prime \prime}$ & NFI & $12-31.2$ & 37 & - & $\mathrm{N}$ \\
\hline GRB 980109 & $00^{\mathrm{h}} 25^{\mathrm{m}} 56^{\mathrm{s}}$ & $-63^{\circ} 01^{\prime} 24^{\prime \prime}$ & WFC & - & - & - & $\mathrm{N}$ \\
\hline GRB 980326 & $08^{\mathrm{h}} 36^{\mathrm{m}} 26^{\mathrm{s}}$ & $-18^{\circ} 53^{\prime} 00^{\prime \prime}$ & WFC & - & - & - & Y \\
\hline GRB 980329 & $07^{\mathrm{h}} 02^{\mathrm{m}} 37^{\mathrm{s}}$ & $+38^{\circ} 50^{\prime} 46^{\prime \prime}$ & NFI & $7-48.6$ & 63.8 & - & Y \\
\hline GRB 980425 & $19^{\mathrm{h}} 35^{\mathrm{m}} 02^{\mathrm{s}}$ & $-52^{\circ} 50^{\prime} 16^{\prime \prime}$ & NFI & $10.2-52.4$ & 52.1 & $\begin{array}{c}161-185 \\
\text { Nov. } 10.75-12\end{array}$ & $\mathrm{SN}(z=0.0085)$ \\
\hline GRB 980515 & $21^{\mathrm{h}} 16^{\mathrm{m}} 44^{\mathrm{s}}$ & $-67^{\circ} 13^{\prime} 05^{\prime \prime}$ & NFI & $10-47.2$ & 49.1 & $218-265$ & No study \\
\hline GRB 980519 & $23^{\mathrm{h}} 22^{\mathrm{m}} 17^{\mathrm{s}}$ & $+77^{\circ} 15^{\prime} 53^{\prime \prime}$ & NFI & $9.7-35.2$ & 78 & - & $\mathrm{Y}$ \\
\hline GRB 980613 & $10^{\mathrm{h}} 18^{\mathrm{m}} 04^{\mathrm{s}}$ & $+71^{\circ} 33^{\prime} 58^{\prime \prime}$ & NFI & $8.6-35.3$ & 61.5 & - & $Y(z=1.1)$ \\
\hline GRB 980703 & $23^{\mathrm{h}} 59^{\mathrm{m}} 07^{\mathrm{s}}$ & $+08^{\circ} 35^{\prime} 06^{\prime \prime}$ & (RXTE) & $22.3-45.6$ & 39.2 & $110.3-132.6$ & $\mathrm{Y}(z=0.97)$ \\
\hline GRB 981226 & $23^{\mathrm{h}} 29^{\mathrm{m}} 37^{\mathrm{s}}$ & $-23^{\circ} 55^{\prime} 45^{\prime \prime}$ & NFI & $6.5-61$ & 89 & 172-191 & $\mathrm{N}$ \\
\hline GRB 990123 & $15^{\mathrm{h}} 25^{\mathrm{m}} 31^{\mathrm{s}}$ & $+44^{\circ} 45^{\prime} 52^{\prime \prime}$ & NFI & $5.8-53.9$ & 81.9 & - & $\mathrm{Y}(z=1.62)$ \\
\hline GRB 990217 & $03^{\mathrm{h}} 02^{\mathrm{m}} 45^{\mathrm{s}}$ & $-53^{\circ} 06^{\prime} 11^{\prime \prime}$ & NFI & $6-44$ & 56.4 & - & $\mathrm{N}$ \\
\hline GRB 990510 & $13^{\mathrm{h}} 38^{\mathrm{m}} 03^{\mathrm{s}}$ & $-80^{\circ} 29^{\prime} 44^{\prime \prime}$ & NFI & $8-44.4$ & 67.9 & - & $Y(z=1.6)$ \\
\hline GRB 990625 & $00^{\mathrm{h}} 26^{\mathrm{m}} 34^{\mathrm{s}}$ & $-32^{\circ} 12^{\prime} 00^{\prime \prime}$ & WFC & - & - & - & No study \\
\hline GRB 990627 & $01^{\mathrm{h}} 48^{\mathrm{m}} 23^{\mathrm{s}}$ & $-77^{\circ} 05^{\prime} 22^{\prime \prime}$ & NFI & $8-39.7$ & 30 & - & $\mathrm{N}$ \\
\hline GRB 990704 & $12^{\mathrm{h}} 19^{\mathrm{m}} 28^{\mathrm{s}}$ & $-03^{\circ} 50^{\prime} 00^{\prime \prime}$ & NFI & $7.5-29.5$ & 37 & $169.8-195$ & $\mathrm{~N}$ \\
\hline GRB 990705 & $05^{\mathrm{h}} 09^{\mathrm{m}} 52^{\mathrm{s}}$ & $-72^{\circ} 08^{\prime} 02^{\prime \prime}$ & WFC & $11-33.8$ & 77.8 & - & $\mathrm{Y}(z=0.86)$ \\
\hline GRB 990712 & $22^{\mathrm{h}} 31^{\mathrm{m}} 49^{\mathrm{s}}$ & $-73^{\circ} 24^{\prime} 24^{\prime \prime}$ & WFC & - & - & - & $\mathrm{Y}(z=0.43)$ \\
\hline GRB 990806 & $03^{\mathrm{h}} 10^{\mathrm{m}} 36^{\mathrm{s}}$ & $-68^{\circ} 07^{\prime} 13^{\prime \prime}$ & NFI & $8-48.9$ & 77.9 & - & $\mathrm{N}$ \\
\hline GRB 990907 & $07^{\mathrm{h}} 31^{\mathrm{m}} 07^{\mathrm{s}}$ & $-69^{\circ} 27^{\prime} 24^{\prime \prime}$ & NFI & $11-11.4$ & 1.1 & - & $\mathrm{N}$ \\
\hline GRB 990908 & $06^{\mathrm{h}} 52^{\mathrm{m}} 53^{\mathrm{s}}$ & $-74^{\circ} 59^{\prime} 17^{\prime \prime}$ & WFC & - & - & - & $\mathrm{N}$ \\
\hline GRB 991014 & $06^{\mathrm{h}} 51^{\mathrm{m}} 02^{\mathrm{s}}$ & $+11^{\circ} 35^{\prime} 37^{\prime \prime}$ & NFI & $13-33.9$ & 36.1 & $258-285.8$ & $\mathrm{~N}$ \\
\hline GRB 991105 & $12^{\mathrm{h}} 03^{\mathrm{m}} 29^{\mathrm{s}}$ & $-67^{\circ} 45^{\prime} 25^{\prime \prime}$ & WFC & - & - & - & $\mathrm{N}$ \\
\hline GRB 991106 & $22^{\mathrm{h}} 24^{\mathrm{m}} 43^{\mathrm{s}}$ & $+54^{\circ} 23^{\prime} 22^{\prime \prime}$ & NFI & $8-26.8$ & 31.6 & - & $\mathrm{N}$ \\
\hline GRB 000210 & $01^{\mathrm{h}} 59^{\mathrm{m}} 17^{\mathrm{s}}$ & $-40^{\circ} 39^{\prime} 17^{\prime \prime}$ & NFI & $7.2-40.2$ & 44.4 & - & $\mathrm{N}(z=0.835)$ \\
\hline GRB 000214 & $18^{\mathrm{h}} 54^{\mathrm{m}} 28^{\mathrm{s}}$ & $-66^{\circ} 27^{\prime} 59^{\prime \prime}$ & NFI & $12-41.5$ & 50.8 & - & $\mathrm{N}(z=0.37-0.47)$ \\
\hline GRB 000528 & $10^{\mathrm{h}} 45^{\mathrm{m}} 09^{\mathrm{s}}$ & $-33^{\circ} 59^{\prime} 01^{\prime \prime}$ & NFI & $12-27.3$ & 26.6 & $78.8-99$ & $\mathrm{~N}$ \\
\hline GRB 000529 & $00^{\mathrm{h}} 09^{\mathrm{m}} 27^{\mathrm{s}}$ & $-61^{\circ} 31^{\prime} 43^{\prime \prime}$ & NFI & $7.4-50.5$ & 34.8 & - & $\mathrm{N}$ \\
\hline GRB 000615 & $15^{\mathrm{h}} 32^{\mathrm{m}} 42^{\mathrm{s}}$ & $+73^{\circ} 47^{\prime} 23^{\prime \prime}$ & NFI & $10-41.6$ & 44.6 & - & $\mathrm{N}$ \\
\hline GRB 000620 & $07^{\mathrm{h}} 35^{\mathrm{m}} 29^{\mathrm{s}}$ & $+69^{\circ} 11^{\prime} 56^{\prime \prime}$ & WFC & - & - & - & $\mathrm{N}$ \\
\hline GRB 000926 & $17^{\mathrm{h}} 04^{\mathrm{m}} 06^{\mathrm{s}}$ & $+51^{\circ} 47^{\prime} 37^{\prime \prime}$ & $(\mathrm{IPN})$ & $48.9-61$ & 19.6 & - & $\mathrm{Y}(z=2.066)$ \\
\hline GRB 001011 & $18^{\mathrm{h}} 23^{\mathrm{m}} 04^{\mathrm{s}}$ & $+50^{\circ} 53^{\prime} 56^{\prime \prime}$ & WFC & - & - & - & $\mathrm{N}$ \\
\hline GRB 001109 & $18^{\mathrm{h}} 30^{\mathrm{m}} 08^{\mathrm{s}}$ & $+55^{\circ} 18^{\prime} 14^{\prime \prime}$ & NFI & $16-37.8$ & 33.2 & $70-106$ & $\mathrm{~N}$ \\
\hline GRB 010213 & $17^{\mathrm{h}} 09^{\mathrm{m}} 22^{\mathrm{s}}$ & $+39^{\circ} 15^{\prime} 36^{\prime \prime}$ & WFC & - & - & - & no study \\
\hline GRB 010214 & $17^{\mathrm{h}} 40^{\mathrm{m}} 56^{\mathrm{s}}$ & $+48^{\circ} 34^{\prime} 52^{\prime \prime}$ & NFI & $6-51.8$ & 83 & - & $\mathrm{N}$ \\
\hline GRB 010220 & $02^{\mathrm{h}} 36^{\mathrm{m}} 59^{\mathrm{s}}$ & $+61^{\circ} 45^{\prime} 57^{\prime \prime}$ & WFC & $15-36$ & 17.2 & - & $\mathrm{N}$ \\
\hline GRB 010222 & $14^{\mathrm{h}} 52^{\mathrm{m}} 12^{\mathrm{s}}$ & $+43^{\circ} 01^{\prime} 00^{\prime \prime}$ & NFI & $8-64$ & 88.3 & - & $\mathrm{Y}(z=1.48)$ \\
\hline GRB 010304 & $21^{\mathrm{h}} 06^{\mathrm{m}} 22^{\mathrm{s}}$ & $+53^{\circ} 12^{\prime} 36^{\prime \prime}$ & WFC & - & - & - & no study \\
\hline GRB 010412 & $19^{\mathrm{h}} 39^{\mathrm{m}} 39^{\mathrm{s}}$ & $+13^{\circ} 37^{\prime} 05^{\prime \prime}$ & WFC & - & - & - & $\mathrm{N}$ \\
\hline GRB 010501 & $19^{\mathrm{h}} 06^{\mathrm{m}} 50^{\mathrm{s}}$ & $-70^{\circ} 10^{\prime} 48^{\prime \prime}$ & WFC & - & - & - & no study \\
\hline GRB 010518 & $10^{\mathrm{h}} 46^{\mathrm{m}} 43^{\mathrm{s}}$ & $-57^{\circ} 47^{\prime} 37^{\prime \prime}$ & WFC & - & - & - & no study \\
\hline GRB 011121 & $11^{\mathrm{h}} 34^{\mathrm{m}} 29^{\mathrm{s}}$ & $-76^{\circ} 01^{\prime} 52^{\prime \prime}$ & NFI & $21.9-65$ & 32.5 & $86.7-120$ & $\mathrm{Y}(z=0.36)$ \\
\hline GRB 011211 & $11^{\mathrm{h}} 15^{\mathrm{m}} 16^{\mathrm{s}}$ & $-21^{\circ} 55^{\prime} 44^{\prime \prime}$ & WFC & - & - & - & $\mathrm{Y}(z=2.14)$ \\
\hline GRB 020321 & $16^{\mathrm{h}} 13^{\mathrm{m}} 05^{\mathrm{s}}$ & $-83^{\circ} 42^{\prime} 35^{\prime \prime}$ & WFC & $6-10.8$ & 6.1 & - & $\mathrm{N}$ \\
\hline GRB 020322 & $18^{\mathrm{h}} 00^{\mathrm{m}} 58^{\mathrm{s}}$ & $+81^{\circ} 06^{\prime} 41^{\prime \prime}$ & NFI & $6-12.4$ & 12.3 & $26.8-33.2$ & Y \\
\hline GRB 020409 & $08^{\mathrm{h}} 45^{\mathrm{m}} 14^{\mathrm{s}}$ & $+66^{\circ} 41^{\prime} 16^{\prime \prime}$ & WFC & - & - & - & $\mathrm{N}$ \\
\hline GRB 020410 & $22^{\mathrm{h}} 06^{\mathrm{m}} 27^{\mathrm{s}}$ & $-83^{\circ} 49^{\prime} 28^{\prime \prime}$ & NFI & $20-27.5$ & 22.8 & $54.3-59.6$ & Y \\
\hline GRB 020427 & $22^{\mathrm{h}} 09^{\mathrm{m}} 21^{\mathrm{s}}$ & $-65^{\circ} 19^{\prime} 42^{\prime \prime}$ & NFI & $11-14.3$ & 6.8 & $60.2-66$ & $\mathrm{~N}$ \\
\hline
\end{tabular}


M. De Pasquale et al.: BeppoSAX GRB X-ray afterglows catalog, Online Material $p 3$

Table 7. Properties of the prompt emission of BeppoSAX Gamma Ray Bursts reported in Table 6 . We indicate the duration and fluence both in the $\mathrm{X}$-ray (2.0-10.0 keV band) and the $\gamma$-ray (40.0-700 keV band). An X following the source name denotes an X-ray-rich GRB or an X-ray flash.

\begin{tabular}{|c|c|c|c|c|c|}
\hline GRB name & $\begin{array}{c}\gamma \text {-ray } \\
\text { duration } \\
(\mathrm{T}, \mathrm{s})\end{array}$ & $\begin{array}{c}\text { X-ray } \\
\text { duration } \\
(\mathrm{T}, \mathrm{s})\end{array}$ & $\begin{array}{c}\gamma \text {-ray } \\
\text { fluence } \\
10^{-7} \mathrm{erg} \mathrm{cm}^{-2}\end{array}$ & $\begin{array}{c}\text { X-ray } \\
\text { fluence } \\
10^{-7} \mathrm{erg} \mathrm{cm}^{-2}\end{array}$ & Ref. \\
\hline GRB 960720 & 8 & 17 & $26 \pm 3$ & $0.8 \pm 0.2$ & $1,2,3$ \\
\hline GRB 970111 & 43 & 60 & $430 \pm 30$ & $16 \pm 1$ & $4,2,3$ \\
\hline GRB 970228 & 80 & 80 & 64.5 & 15.4 & 5 \\
\hline GRB 970402 & 150 & 150 & $82 \pm 9$ & $4.7 \pm 1.5$ & 2 \\
\hline GRB 970508 & 15 & 29 & 14.5 & 5.3 & 5 \\
\hline GRB 971214 & 35 & 35 & 64.9 & 2.34 & 5,3 \\
\hline GRB 971227 & 7 & 7 & $6.6 \pm 0.7$ & 1 & 6,3 \\
\hline GRB 980109 & 20 & 20 & $32.3 \pm 3$ & - & 3,7 \\
\hline GRB 980326 & 9 & 9 & $7.5 \pm 1.5$ & $2 \pm 0.3$ & 5,3 \\
\hline GRB 980329 & 58 & 68 & $650 \pm 50$ & $9.7 \pm 0.7$ & 5 \\
\hline GRB 980425 & 31 & 40 & $28.5 \pm 5$ & $7.8 \pm 0.2$ & 2,3 \\
\hline GRB 980515 & 15 & 20 & $23 \pm 3$ & - & 7,3 \\
\hline GRB 980519 & 30 & 190 & $81 \pm 5$ & 18 & $8,9,3$ \\
\hline GRB 980613 & 50 & 50 & 9.9 & 2.3 & 5,3 \\
\hline GRB 981226X & 20 & 260 & $4 \pm 1$ & $5.7 \pm 1$ & 10,3 \\
\hline GRB 980703 & 90 & - & $300 \pm 100$ & - & 11 \\
\hline GRB 990123 & 100 & 100 & 1790 & 22.9 & 5,3 \\
\hline GRB 990217 & 25 & 25 & $12.7 \pm 1.5$ & - & 7,3 \\
\hline GRB 990510 & 75 & 80 & 181 & 17.9 & 3,5 \\
\hline GRB 990625 & 11 & 11 & - & - & 3 \\
\hline GRB 990627 & 28 & 60 & - & $\sim 15$ & 3,12 \\
\hline GRB 990704X & 23 & 40 & $10 \pm 1$ & $15 \pm 0.8$ & 13,3 \\
\hline GRB 990705 & 42 & 45 & 423 & 22.5 & 5,3 \\
\hline GRB 990712 & 30 & 30 & $65 \pm 3$ & 28.6 & 5,3 \\
\hline GRB 990806 & 30 & 30 & $\sim 42$ & $\sim 2.5$ & 14,3 \\
\hline GRB 990907 & 1 & 220 & - & - & 3 \\
\hline GRB 990908 & 50 & 130 & - & - & 3 \\
\hline GRB 991014 & 3 & 10 & $9 \pm 1$ & 1 & $15,16,3$ \\
\hline GRB 991105 & 13 & 40 & - & - & 3 \\
\hline GRB $991106^{a}$ & - & 5 & $<1.2^{b}$ & & 17 \\
\hline GRB 000210 & 10 & 115 & $610 \pm 20$ & $\sim 15$ & 18,3 \\
\hline GRB 000214 & 115 & 100 & 61.7 & 11.6 & 5,3 \\
\hline GRB 000528 & 80 & 120 & $14.4 \pm 0.4$ & - & 19,20 \\
\hline GRB 000529 & 14 & 30 & - & - & 3 \\
\hline GRB 000615X & 12 & 120 & $9.8 \pm 0.9$ & $17 \pm 1$ & 21,3 \\
\hline GRB 000620 & 15 & 20 & - & - & 3 \\
\hline GRB 001011 & 31 & 60 & - & - & 3 \\
\hline GRB 001109 & 60 & 65 & $49.7 \pm 1.9$ & $6.4 \pm 0.33$ & 22,3 \\
\hline GRB 010213 & 23 & 25 & - & - & 3 \\
\hline GRB 010214 & 15 & 30 & $45 \pm 0.8$ & $2 \pm 0.3$ & 23 \\
\hline GRB 010220 & 40 & 150 & - & - & 3 \\
\hline GRB 010222 & 170 & 280 & 753 & 95 & 5,3 \\
\hline GRB 010304 & 15 & 24 & - & - & 3 \\
\hline GRB 010501 & 37 & 41 & - & - & 3 \\
\hline GRB 010412 & 74 & 90 & - & - & 3 \\
\hline GRB 010518 & 25 & 30 & - & - & 3 \\
\hline GRB 011121 & 105 & 100 & $1000 \pm 20$ & $140 \pm 3$ & 24,3 \\
\hline GRB 011211 & 400 & 400 & $37 \pm 4$ & $11 \pm 1$ & 24,3 \\
\hline GRB 020321 & 70 & 90 & 30 & 0.9 & 25,3 \\
\hline
\end{tabular}


Table 7. continued.

\begin{tabular}{lccccc}
\hline \hline GRB name & $\begin{array}{c}\gamma \text {-ray } \\
\text { duration } \\
(\mathrm{T}, \mathrm{s})\end{array}$ & $\begin{array}{c}\text { X-ray } \\
\text { duration } \\
(\mathrm{T}, \mathrm{s})\end{array}$ & $\begin{array}{c}\gamma \text {-ray } \\
\text { fluence } \\
10^{-7} \mathrm{erg} \mathrm{cm}^{-2}\end{array}$ & $\begin{array}{c}\text { X-ray } \\
\text { fluence } \\
10^{-7} \mathrm{erg} \mathrm{cm}^{-2}\end{array}$ & Ref. \\
\hline GRB 020322 & 15 & 50 & - & - & 3 \\
GRB 020409 & 40 & 60 & - & - & 3 \\
GRB 020410 & 1800 & $>1290$ & $\sim 290$ & $>47$ & 26,3 \\
GRB 020427X & - & 60 & $<2.9$ & $3.7 \pm 0.3$ & 27,3 \\
\hline
\end{tabular}

${ }^{a}$ Perhaps not a GRB. See Cornelisse et al. (2000).

${ }^{b}$ Conservative $3 \sigma$ upper limit based on GCN 448.

References: 1: Piro et al. (1998a), 2: Frontera et al. (2000a), 3: Frontera et al. (2004), 4: Feroci et al. (1998), 5: Amati et al. (2002), 6: Antonelli et al. (1999), 7: Amati et al. (1999), 8: Nicastro et al. (1999), 9: in't Zand et al. (1999), 10: Frontera et al. (2000b), 11: Amati et al. (1998), 12: Muller et al. (1999b), 13: Feroci et al. (2001), 14: Montanari et al. (2002), 15: Tassone et al. (1999), 16: in't Zand et al. (2000b), 17: Gandolfi et al. (1999), 18: Piro et al. (2002), 19: Guidorzi et al. (2000), 20: in't Zand et al. (2000a), 21: Nicastro et al. (2001), 22: Guidorzi et al. (2003), 23: Guidorzi et al. (2003), 24: Piro et al. (2005), 25: in't Zand et al. (2004), 26: Nicastro et al. (2004), and 27: Amati et al. (2004).

Note 1: When not available, values of 2-10 keV fluences have been calculated from the $2-26 \mathrm{keV}$ fluences and assuming the spectral parameters reported in the references.

Note 2: The X-ray and $\gamma$ fluences reported by Amati et al. (2002) have been obtained by reporting the parameters of the WFC and GRBM spectra fit at $z=0$ (see Table 2 of the same article). 
M. De Pasquale et al.: BeppoSAX GRB X-ray afterglows catalog, Online Material p 5
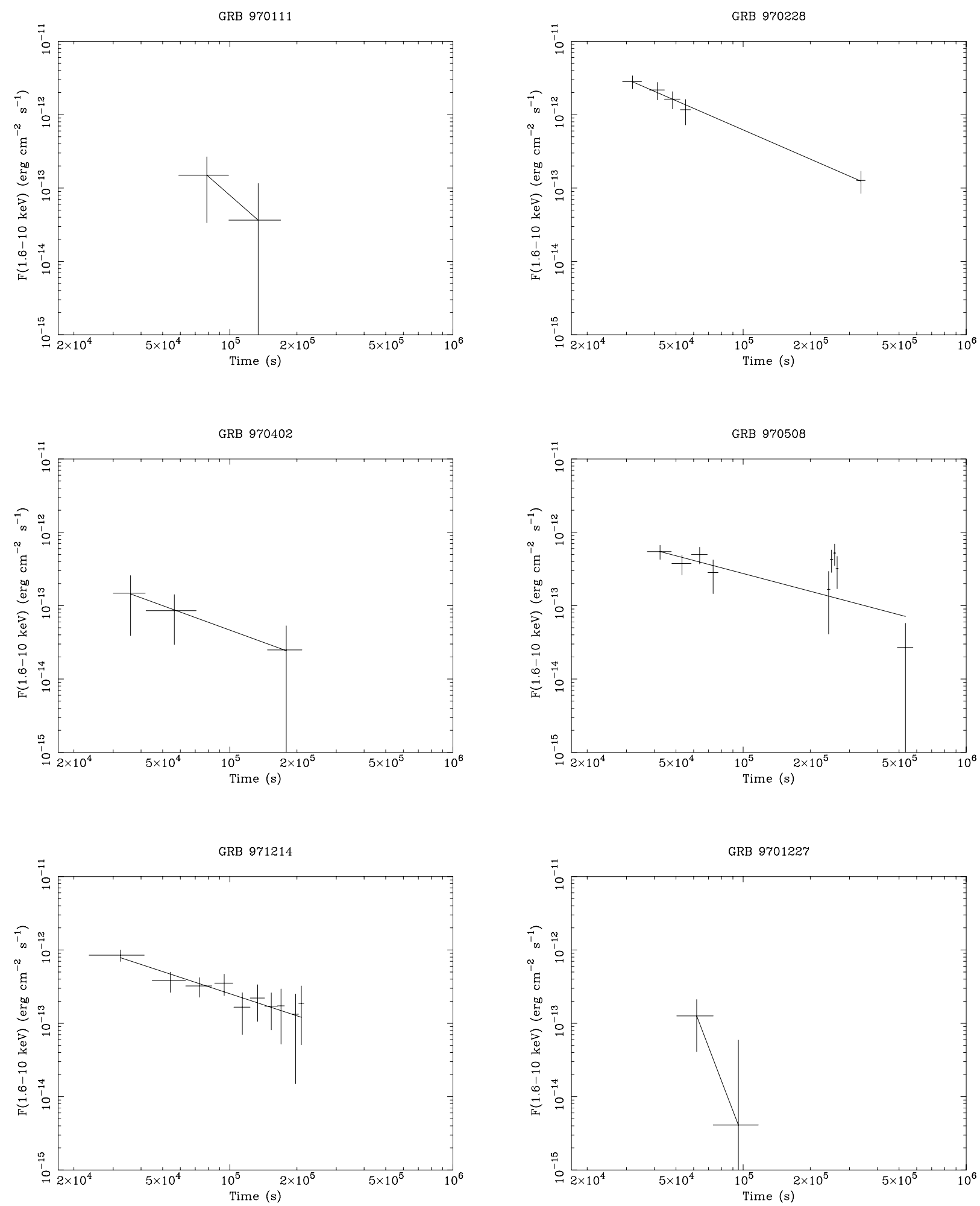

Fig. 12. X-ray lightcurves of the afterglows observed by beppoSAX in the $1.6-10 \mathrm{keV}$ band. 
M. De Pasquale et al.: BeppoSAX GRB X-ray afterglows catalog, Online Material p 6
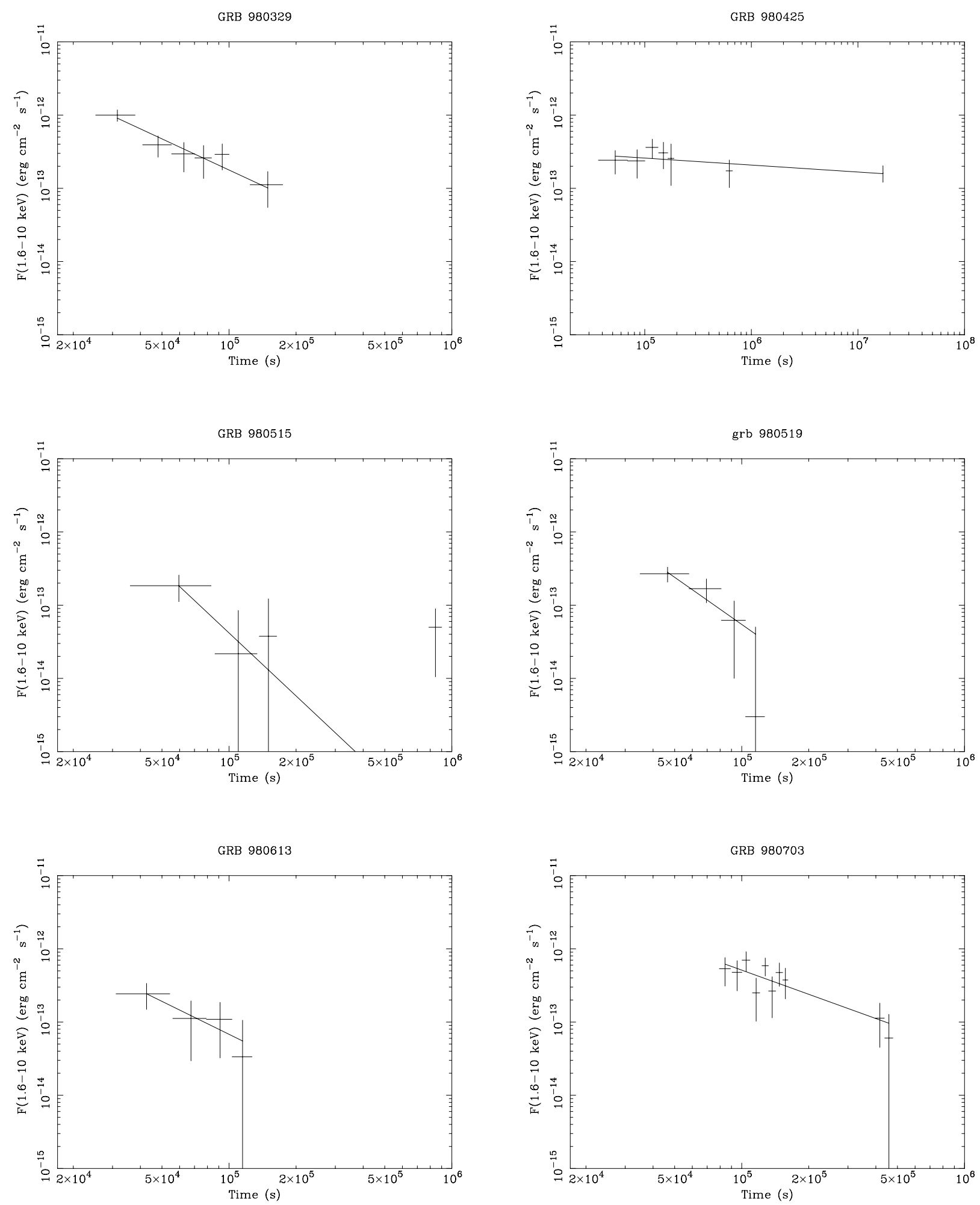

Fig. 12. continued. 
M. De Pasquale et al.: BeppoSAX GRB X-ray afterglows catalog, Online Material $p 7$
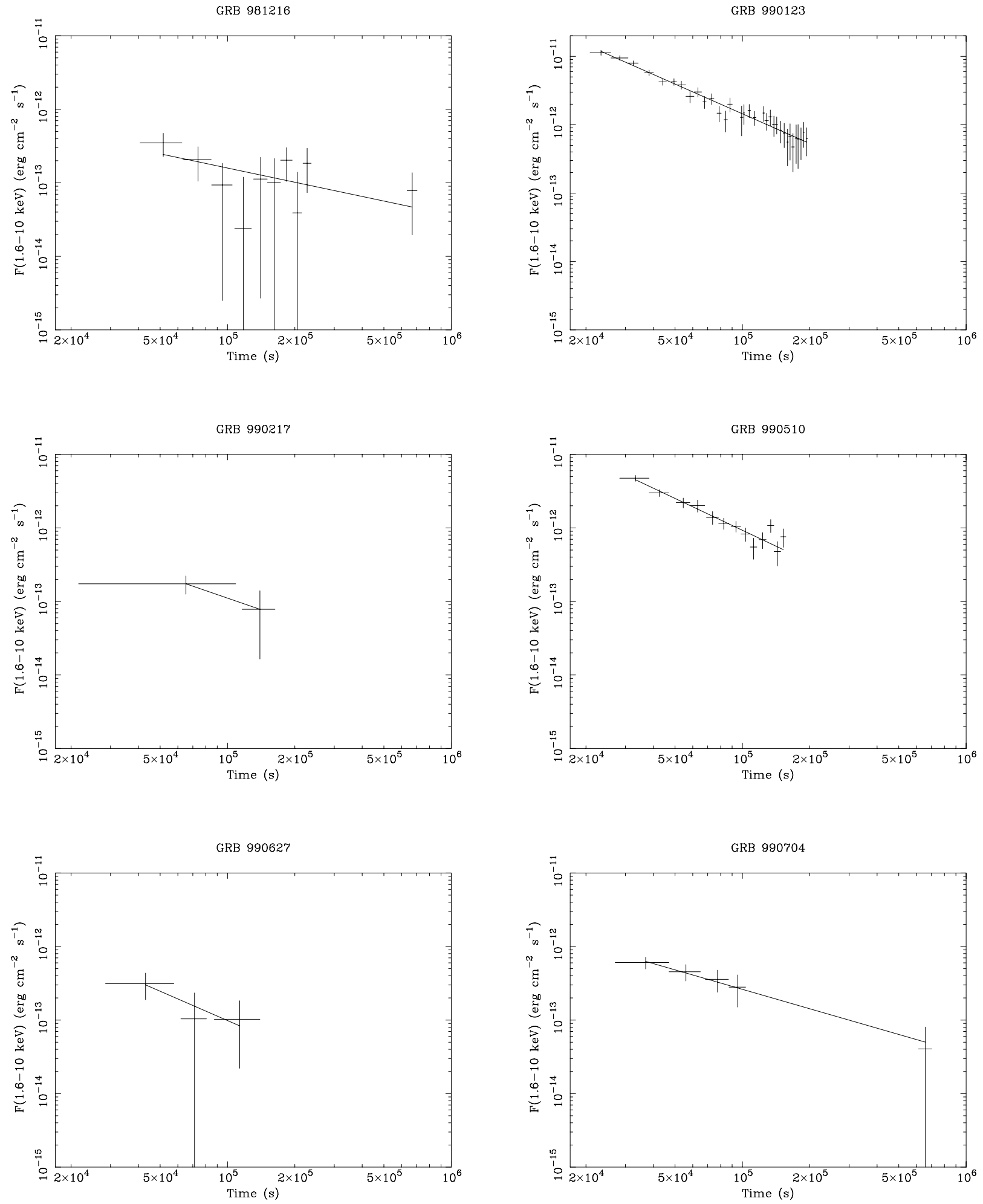

Fig. 12. continued. 
M. De Pasquale et al.: BeppoSAX GRB X-ray afterglows catalog, Online Material $p 8$
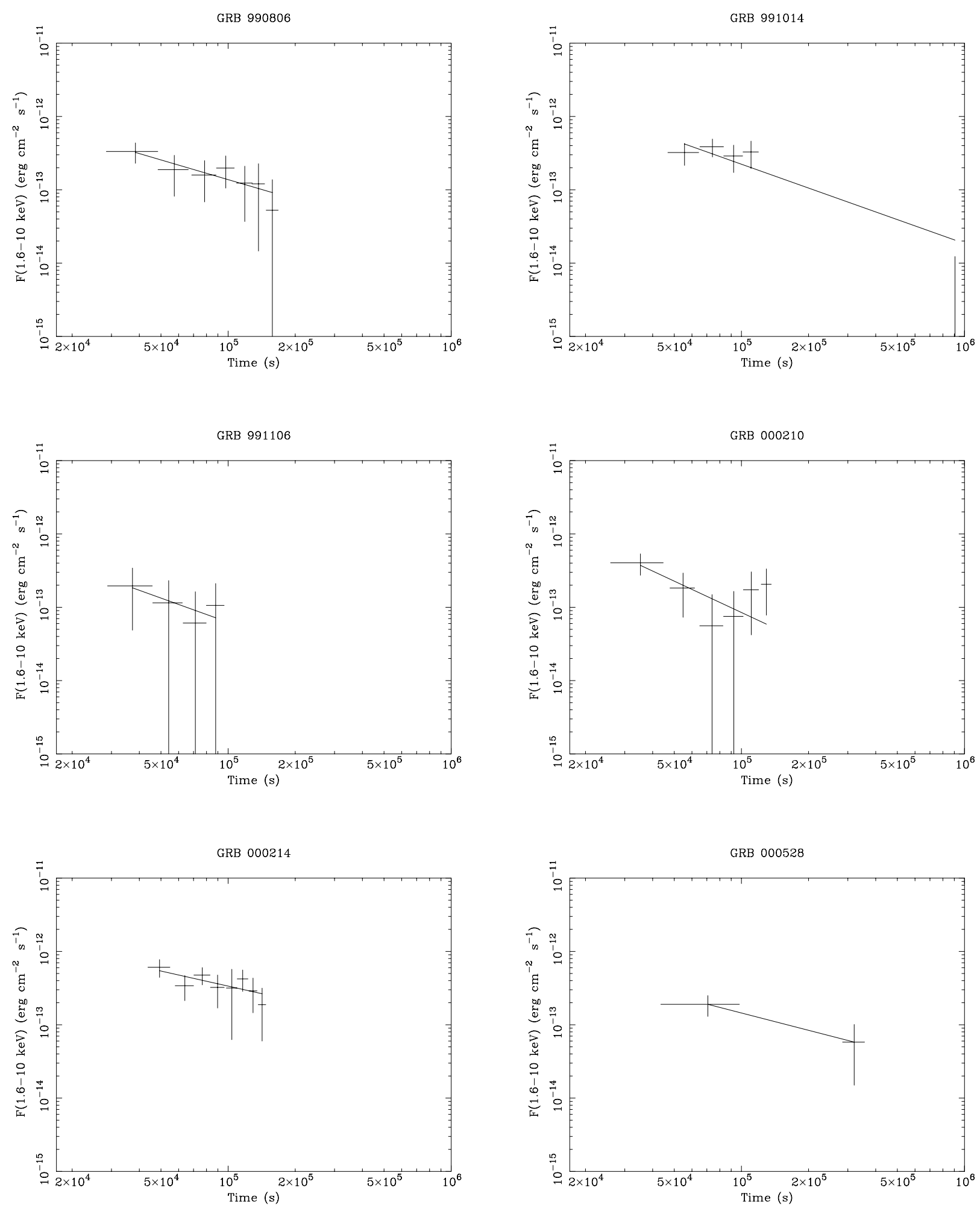

Fig. 12. continued. 
M. De Pasquale et al.: BeppoSAX GRB X-ray afterglows catalog, Online Material p 9
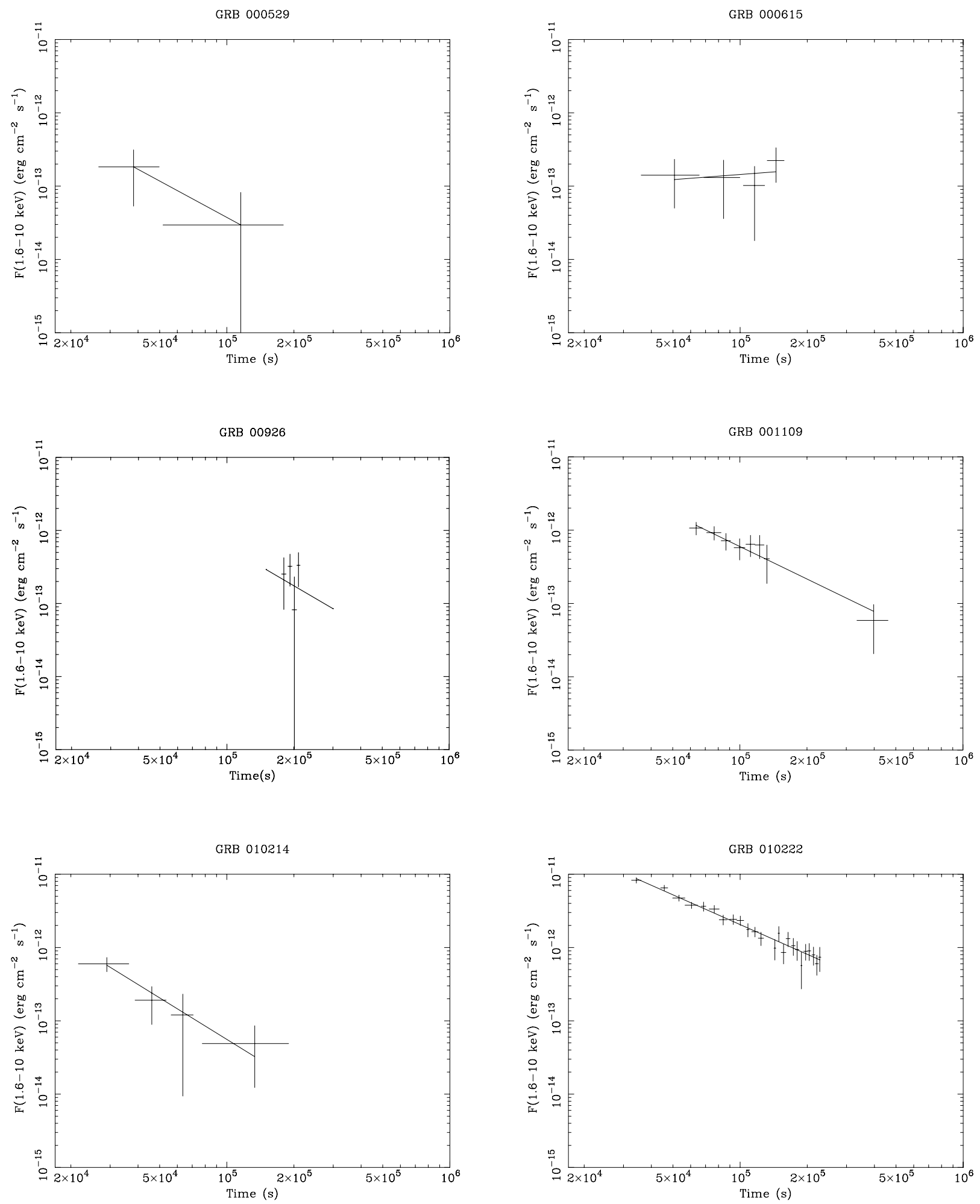

Fig. 12. continued. 
M. De Pasquale et al.: BeppoSAX GRB X-ray afterglows catalog, Online Material p 10
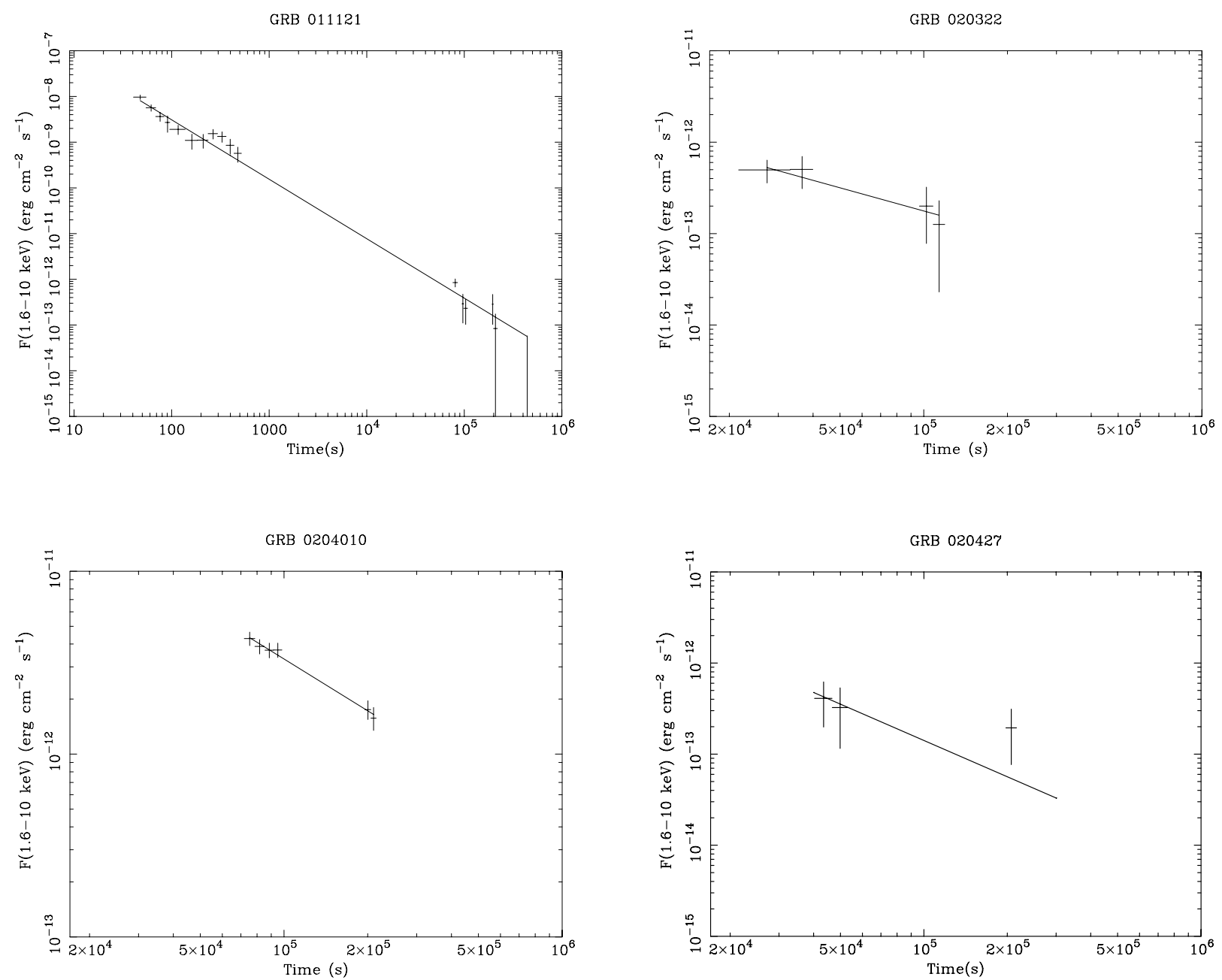

Fig. 12. continued. 